\title{
Training Schrödinger's cat: quantum optimal control
}

\section{Strategic report on current status, visions and goals for research in Europe}

Steffen J. Glaser ${ }^{1}$, Ugo Boscain ${ }^{2}$, Tommaso Calarco ${ }^{3}$, Christiane P. Koch ${ }^{4}$, Walter Köckenberger ${ }^{5}$, Ronnie Kosloff ${ }^{6}$, Ilya Kuprov ${ }^{7}$, Burkhard Luy ${ }^{8}$, Sophie Schirmer ${ }^{9}$, Thomas Schulte-Herbrüggen ${ }^{1}$, Dominique Sugny ${ }^{10,11}$, and Frank K. Wilhelm², a

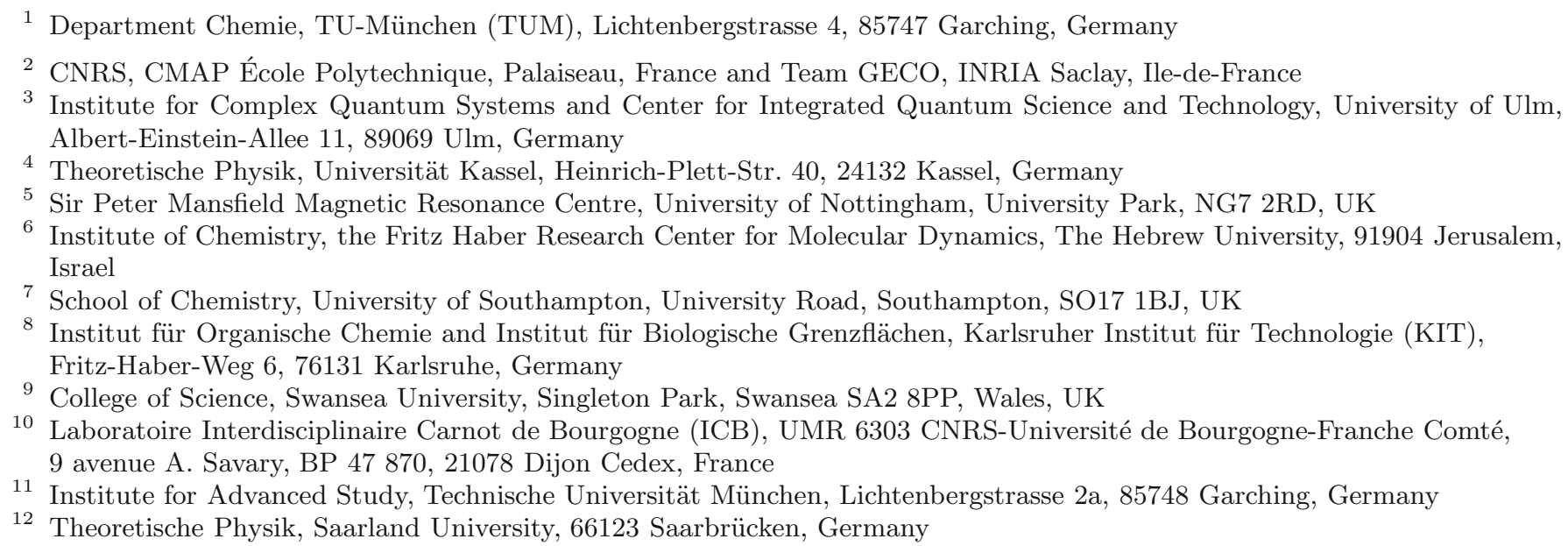

Received 7 August 2015 / Received in final form 22 September 2015

Published online 17 December 2015

(c) The Author(s) 2015. This article is published with open access at Springerlink.com

\begin{abstract}
It is control that turns scientific knowledge into useful technology: in physics and engineering it provides a systematic way for driving a dynamical system from a given initial state into a desired target state with minimized expenditure of energy and resources. As one of the cornerstones for enabling quantum technologies, optimal quantum control keeps evolving and expanding into areas as diverse as quantumenhanced sensing, manipulation of single spins, photons, or atoms, optical spectroscopy, photochemistry, magnetic resonance (spectroscopy as well as medical imaging), quantum information processing and quantum simulation. In this communication, state-of-the-art quantum control techniques are reviewed and put into perspective by a consortium of experts in optimal control theory and applications to spectroscopy, imaging, as well as quantum dynamics of closed and open systems. We address key challenges and sketch a roadmap for future developments.
\end{abstract}

\section{Foreword}

The authors of this paper represent the QUAINT consortium, a European Coordination Action on Optimal Control of Quantum Systems, funded by the European Commission Framework Programme 7, Future Emerging Technologies FET-OPEN programme and the Virtual Facility for Quantum Control (VF-QC). This consortium has considerable expertise in optimal control theory and its applications to quantum systems, both in existing areas, such as spectroscopy and imaging, and in emerging quantum technologies, such as quantum information processing, quantum communication, quantum simulation

\footnotetext{
a e-mail: fwm@lusi.uni-sb.de
}

and quantum sensing. The list of challenges for quantum control has been gathered by a broad poll of leading researchers across the communities of general and mathematical control theory, atomic, molecular-, and chemical physics, electron and nuclear magnetic resonance spectroscopy, as well as medical imaging, quantum information, communication and simulation. 144 experts in these fields have provided feedback and specific input on the state of the art, mid-term and long-term goals. Those have been summarized in this document, which can be viewed as a perspectives paper, providing a roadmap for the future development of quantum control. Because such an endeavour can hardly ever be complete (there are many additional areas of quantum control applications, such as spintronics, nano-optomechanical technologies etc.), this roadmap 
is designed as a living document that is available at the homepage of the VF-QC, [1], where additional aspects, as well as new developments and ideas, will be included.

\section{Introduction}

It is control that turns scientific knowledge into technology. The general goal of quantum control is to manipulate dynamical processes at the atomic or molecular scale, typically using external electromagnetic fields. The objective of quantum optimal control is to devise and implement shapes of pulses of external fields, or sequences of such pulses, that accomplish a given task in a quantum system in the best way possible. Quantum control builds on a variety of theoretical and technological advances, from the fields of mathematical control theory and numerical mathematics to better electronic devices, such as arbitarywaveform generators with sub-nanosecond time resolution or stronger magnetic fields.

The challenge of manipulating nature at the quantum level has a huge potential for current and future applications. Quantum systems and processes cover a wide range from atomic and molecular physics, chemistry and materials (such as semiconductors, superconductors) to biosystems and medicine. Useful applications range from magnetic resonance imaging and spectroscopy and the precise control of chemical reactions to emerging "second generation" quantum technologies. Quantum optimal control is a part of the effort to engineer quantum technologies from the bottom up, and many striking examples of surprising and non-intuitive - but extremely efficient and robust quantum control techniques have been discovered in recent years. Examples of important current applications are precise measurement of magnetic fields with nanometer resolution using NV centers in diamond, state engineering in Bose-Einstein condensates and high-fidelity quantum gates in superconducting quantum processors. In a similar way to the first generation of quantum-based technologies that brought forward semiconductor transistors, lasers, magnetic resonance imaging and spectroscopy, the currently emerging second generation of quantum technologies based on superposition, entanglement and many-body quantum states are expected to generate new and disruptive technologies - spintronic devices, quantum metrology, quantum computing technology, as well as novel instruments for elucidating chemical reaction dynamics and material properties. Quantum control is thus a strategic cross-sectional field of research, enabling and leveraging current and future quantum technology applications.

While the instrumental details of manipulating the behavior of all those systems may differ (lasers, radio waves etc.), the control, identification and system design problems encountered share the same mathematical formalism that is distinct from classical control theory. Advancing quantum control therefore requires bringing together researchers from different application areas to forge a community, creating a common language and identifying common challenges. Further development of this field of research offers many beneficial effects for today's and tomorrow's society, related to health, secure communication, accurate navigation systems, efficient harvesting of solar power, the search for resources, efficient energy storage and transportation, quantum machines, precision sensing and monitoring of the environment.

The paper is organized as follows: Section 2 is focussed on mathematical optimal control theory. It is followed by a description of the state of the art, as well as mid-and long-term perspectives for quantum control applications in atomic, molecular, and chemical physics (Sect. 3), magnetic resonance (Sect. 4) and quantum information and communication (Sect. 5). Prospects for applications and commercial exploitation are outlined in Section 6 and conclusions are given in Section 7.

\section{General aspects and mathematics of optimal control}

The recent advances in quantum control, by now recognized to be essential for continued development of quantum technologies (see, e.g., [2,3]) and reviewed in Sections 3 to 5 below, are based on powerful tools from mathematical control theory [4-13]. As it often happens on the interface between mathematics and physics, mathematical concepts not only prove fruitful for the solution of physical problems, but in turn specific physical features require further mathematical development. In the context of quantum control, these are, for example, entanglement and the nature of quantum measurements. Eventually, a new research domain, mathematical quantum control theory [14-23] has emerged (see also [11,24], Sect. 19.3).

In general, quantum control theory needs to answer two fundamental questions: that of controllability (i.e., what control targets are accessible, cf. Section 2.1) and that of control design (i.e. how can a target be reached). Approaches to control design can be open-loop or closedloop. In the latter case, the specific nature of quantum measurements needs to be taken into account. This is covered in Section 2.3. The remaining control design approaches are reviewed in Section 2.2. Open-loop techniques include approaches based on the Pontryagin maximum principle [4] with solutions obtained analytically (Sect. 2.2.1) or numerically (Sect. 2.2.2). Optimal control does not make any assumptions about the system and experimental constraints and robustness requirements can be fully taken into account, i.e. it is generally applicable. However in some cases, adiabatic control and its variants (cf. Sect. 2.2.3) can provide a more straight forward approach for solving robustness issues and implementing constraints.

\subsection{Controllability and simulability}

\subsubsection{State of the art}

Controllability analysis determines whether a quantum system can be brought from any given initial state to 
any desired target state, or, more generally, from a given set of initial states to any set of target states. Adapting results from classical linear control systems $[7,8]$ to bilinear systems with a non-switchable drift term, a rigorous Lie-framework was developed for closed systems [12,25-29]. Based on this work, for quantum systems with finite dimension, controllability by now is well understood $[14,18,20,30-33]^{1}$. Different notions of controllability have been introduced for pure states, mixed states, and evolution operator dynamics [34]. The main controllability test is based on the rank of the dynamical Lie algebra, which is generated by the drift and the different control Hamiltonians. The difficulty of using the rank condition in large systems has motivated a geometric approach based on graph theory [35], yielding eventually a complete set of symmetry criteria for controllability [20].

In infinite-dimensional systems, the mathematics is much more intricate and the few existing results are confined to quantum systems with a discrete spectrum. An important result is a general obstruction property to exact controllability $[33,36,37]$. This was recently amended by positive results about exact [38-40] and approximate controllability $[32,41-44]$, based on Galerkin techniques. For the specific case of a generalized Jaynes-Cummings model, i.e., several two-level systems coupled to a harmonic oscillator, symmetry methods were used to assess controllability $[45,46]$.

Simultaneous controllability concerns the control of a continuum of finite dimensional quantum systems by only a few control fields. This is also known as ensemble controllability. Approximate and exact controllability results have been obtained recently in this direction [47-50]. This analysis is important for designing control fields which are robust to experimental imperfections [51-56]. Adiabatic techniques could be very useful to this purpose, see Section 2.2.3.

In addition to experimental imperfections and fluctuations, decoherence may pose an obstacle to control. For open quantum systems, the control field usually cannot fully compensate dissipation, as rigorously shown for the case of Markovian dynamics [57]. These results for Markovian dynamics were recently generalized to a complete Lie semi-group picture [58,59]. In contrast, controllability of systems with non-Markovian dynamics presents by and large uncharted territory [60]. Reachability is expected to be larger for non-Markovian dynamics since nonMarkovianity implies information back-flow from the environment to the system [61]. Indeed, an explorative study showed non-Markovian map synthesis to be stronger than its restriction to the Markovian case (as anticipated in Ref. [62]). In contrast, and surprisingly so, for the simpler problem of state transfer under swichable Markovian noise this is not the case [63].

Finally, even if a system (A) is not fully controllable, its controlled dynamics may still suffice to generate a desired effective evolution as brought about by another quantum dynamical system (B). This is the paradigm of finite-

\footnotetext{
${ }^{1}$ Note that only the standard finite-dimensional bilinear situation (as in the Schrödinger equation) is discussed here.
}

dimensional quantum simulation. Adapting the tools from controllability analysis, one readily sees that system A can simulate system B if the system algebra of A encompasses that of system B. A recent generalisation of the results in reference [20] provides a complete set of symmetry criteria together with an algorithm for deciding the simulability on system-algebraic grounds [64]. Resorting to exactly solvable problems may help in this case [65].

\subsubsection{Mid-term prospects: goals and challenges}

The major challenge is to better understand controllability in open quantum systems. For infinite-dimensional open systems with a discrete spectrum that undergo Markovian evolution, a rigorous understanding is nontrivial, but may be pursued by extending the standard LieGalerkin techniques to non-unitary evolution. With the final goal of identifying sufficient conditions for approximate controllability of open systems analogous to closed systems $[32,41,43]$, a mid-term perspective is to perform a sound regularity analysis of a well-posed and defined mathematical control problem.

No rigorous controllability analysis so far has tackled open quantum systems which undergo non-Markovian dynamics. From a control point of view, non-Markovianity may be connected to beneficial aspects of the systemenvironment interaction, whereas the detrimental part is linked to those Markovian processes that cannot be remedied - an aspect important not only for controllability, but also for dissipative state engineering and quantum memories. Along similar lines, projective measurements may entail Zeno-type dynamics exploring directions that do not show up in the unobserved system [66].

Even for closed quantum systems, several highly relevant questions are still open. These include the exact or approximate controllability of the Schrödinger equation with mixed or continuous spectrum. This question is important because it covers dissociation and ionization processes, the control of which is a major goal in chemical physics, cf. Section 3.

Beyond controllability, a precise description of the reachable set (both in the closed and in the open case) and upper bounds on minimal time to reach target states are largely unknown. Also, a universal estimate for the time to control finite-dimensional quantum systems (with necessary drift) is still an open challenge.

\subsection{Control design}

\subsubsection{Geometric optimal control - State of the art}

Optimal control theory can be viewed as a generalization of the calculus of variations for problems with dynamical constraints. Its modern version was born with Pontryagin's Maximum Principle in the late 1950's [4], which generalises the classical Euler-Lagrange equations. Its development was boosted by the use of Kalman filters $[7,67]$ in the Apollo programme. It is now a key tool in many applications, including quantum mechanics. 
Solving an optimal control problem means finding a control law (e.g. a pulse sequence), such that the corresponding system trajectory satisfies given boundary conditions and equation of motion and minimizes a cost criterion, such as the energy or duration of the control. Pontryagin's Maximum Principle can be formulated in terms of Hamiltonian-like equations. In a typical workflow, one first obtains extremal trajectories by solving these equations. In the second step, one selects among the extremals those which minimize the cost. Although looking straightforward, the practical use of Pontryagin's Maximum Principle is far from trivial. Apart from integrating the Hamiltonian equations, the main difficulties are the presence of abnormal and singular extremals [68] and the problem of selection of optimal trajectories. For this reason methods from differential geometry and numerical analysis are often required. The latter are described in Section 2.2.2.

If the system is sufficiently simple, for example lowdimensional, the optimal control problem may be solved analytically. Typically, this requires understanding of the geometry of the control problem from which one can deduce the structure of the optimal solution, a proof of global optimality and physical limits, such as the minimal time to reach the target [5]. Mathematical tools that were developed recently [11-13,68-70] could tackle problems of increasing difficulty, including fundamental control problems for closed [14,71-75] and open quantum systems [76-81]. This method is able to treat quantum control problems ranging from two and three level quantum systems or two and three coupled spins to two-level dissipative quantum systems with dynamics governed by the Lindblad equation. Singular optimal arcs have been used to derive control fields in some example quantum systems [77,82-87]. This approach can also be advantageously combined with numerical optimization techniques in order to manipulate more complicated systems [88].

The Cartan decomposition method also turned out to be an efficient tool for the control of spin systems [89-91]. This decomposition leads to a reduction of the state space dimensionality and may enable its geometric description.

\subsubsection{Numerical optimal control - State of the art}

If the set of control equations resulting from the maximum principle cannot be solved analytically, numerical optimal control theory provides a viable alternative. The available techniques include (i) gradient ascent algorithms [92] (which can be extended to second-order quasi-Newton and Newton methods [93-95]) and (ii) Krotov-type methods [96-98], also permitting this extension [99]. The main difference between these two approaches is that the control is updated (or replaced) for all times simultaneously in case (i) and sequentially in case (ii), which implies different convergence properties. The algorithms are comparatively easy to use and several program packages include optimal control modules, e.g. SIMPSON [100], SPINACH [101], DYNAMO [94], and QuTiP [102]. Modifications to account for experimental imperfections and limitations and to ensure robustness of the solution have been introduced [53,103-111], and numerical optimal control theory has been extended to open quantum systems [112-117].

It is also possible to restrict the control solution to a predefined analytical form. In this case, the control only depends on a limited set of parameters which are optimized [118-120]. Applications of numerical optimal control are discussed below in Sections 3 to 5 but by now have grown too numerous for a complete bibliography.

A caveat for numerical algorithms that are derived from the maximum principle is that they require one to solve the system's equations of motion many times, at least twice per iteration step. This potentially hampers application to high-dimensional quantum systems. So far, three approaches have been pursued to cope with this issue. (i) optimization of the state space representation: for the high-temperature regime of ensemble NMR spectroscopy, the SPINACH package [101] uses state propagation with correlations limited to pertinent short-range ones (thus leading to efficient truncation of the underlying spin Lie algebra) for simulating unprecedentedly large spins systems such as entire biomolecules [121] with striking precision. (ii) gradient-free optimization: for many-body quantum systems, the chopped random basis (CRAB) method allows one to generate a parametrization of the control sequence and interfaces the tensor compression of the time-dependent density matrix renormalization group with parameter optimization $[122,123]$. CRAB is part of the QuTIP package [102]. The number of propagations may be significantly reduced compared to gradientbased optimization, provided a small basis is sufficient to represent the control. (iii) local control theory: it determines the control field from instantaneous dynamical properties of the system by requiring monotonic increase or decrease of a performance index [124,125]. Under wellestablished conditions [126], the system converges asymptotically towards the target. Local controls are reminiscent of the closed-loop Lyapunov method in stabilization, and correspondingly the approach has also been termed tracking. Due to the inherent nature of the quantum measurement process, this approach has been transformed into an open-loop control law in the quantum world. In this approach, the equation of motion needs to be solved only once.

Mathematical quantum optimal control theory shows how to design optimal control fields and describes under which conditions they exist $[30,127]$. It does, however, not investigate the complexity inherent to the search. First results on the search complexity [128] have been derived from an information theoretical analysis of quantum optimal control: indeed, connecting the classical information carried by the control field and the size of the effective Hilbert space explored by the dynamics, the (smoothed) complexity of the search is found to be polynomial and the minimal achievable error as well as the minimal duration of the optimal control field as a function of its bandwidth are bounded. The latter is a direct consequence of the finite classical channel capacity of the control field, similarly to the quantum speed limit that arises from 
the energy-time uncertainty relation. These results can be generalized to the presence of noise [128]. The search complexity is related to the description of the control landscape which specifies the control objective as a function of the control variables. Different results on the structure of the control landscape have been established recently [129-132]. One of the main claims has been that, provided there are no constraints on the control, the control landscape does not contain local traps in all controllable closed and some specific open quantum systems [17]. This proof has recently been contested and is still the object of a lively debate [131-133]. Once constraints are present, such as finite control field amplitude, this may induce traps in the control landscape $[134,135]$. Whether and how imposing robustness of the control influences the control landscape is an open question.

\subsubsection{Control via adiabatic dynamics - State of the art}

While optimal control theory is a general approach that is particularly well suited for identifying fast controls, the target can also be tackled by adiabatic techniques [136-142]. The most widely known among these is Stimulated Raman Adiabatic Passage (STIRAP) [136]. Adiabatic techniques usually employ a sequence of very intense pulses over a comparatively long timescale which enforces adiabatic following of the system dynamics. The pulses can be frequency chirped according to the structure of the energy levels. Such processes are inherently robust to small variations of laser or system parameters and thus well-suited to open-loop control [50]. A main drawback of adiabatic control is the total time and energy requirements which cannot always be met in an experiment.

To address this issue, shortcuts to adiabaticity were developed [143]. This approach can be viewed as an inverse engineering technique based on Lewis-Riesenfeld invariants. It has been applied to a variety of quantum systems [144-147] for which such an invariant exists. Even for an ensemble of quantum systems, analytical solutions based on this technique are not out of reach [146]. Similarly to enforcing robustness in ensemble optimization using optimal control $[53,56]$, the basic idea consists in selecting among a family of (exact) solutions the ones that are effectively robust to some extent to variations of the system parameters.

\subsubsection{Mid-term prospects: goals and challenges for control design}

As with controllability analysis, the major challenge for control design is posed by open quantum systems because most systems of interest interact with their environment to a non-negligible extent. An important challenge is the extension of the standard adiabatic and shortcut techniques to open quantum systems. Beyond some preliminary results $[148,149]$, there are many open questions, for example, on how to generalize Lewis-Riesenfeld invariants to open system dynamics.
All open-loop control approaches discussed above assume sufficient knowledge of the quantum system. Correspondingly, for open quantum systems, system identification needs to include dissipative parameters. A first step to combine quantum control with implicit learning about the system parameters is the ADHOC technique [150].

Decoherence in open quantum systems can also be used as a resource in what has become known as dissipative state engineering [151-154]. Optimal control theory allows for tackling dissipative state engineering for quantum systems that are too complex for manual design of the driven-dissipative dynamics. This naturally includes incorporation of the noise as additional optimization parameter $[63,155]$. When added to coherent controls, timedependent Markovian noise (amplitude-damping) enables the control system to transform any initial state into any desired target state [63]. It can be easily integrated into toolboxes like DYNAMO [94], yet the implementation in realistic settings remains unexplored.

On the algorithmic level, the basics of numerical quantum optimal control are well established for both Markovian and non-Markovian open systems, as described above. The main challenge is the efficient numerical modeling of increasingly complex dynamics. A promising route is provided by stochastic methods that only require propagation of (several) pure states to unravel the true dynamics $[115,156,157]$. These methods are applicable to systems interacting with an environment or subjected to measurements that can be modelled by a stochastic process. An open question in this context is to find the most efficient way to control these systems such that the control is robust with respect to the stochastic parameter [158].

In view of numerical optimal control theory as an openloop technique, integration with applications is a crucial issue that has only very partially been addressed. In order to make the theory more useful for specific experiments, the gap between theory and experiments needs to be bridged. Despite recent progress, a systematic and efficient algorithm addressing this issue is still lacking. One option consists in combining open-loop optimal algorithms with closed-loop control techniques in order to reduce their respective drawbacks [150]. Using these two tools cooperatively is expected to provide flexible quantum control. A current challenge is to improve the computational speed and accuracy of the algorithms. A promising approach to this end is the combined use of numerical and geometric optimal control methods [88]. Analogously to open quantum systems, improving the computational speed will be an important prerequisite to attack control problems of increasing complexity, representing experimental settings in a realistic way. Eventually, the goal is to enable the problem-adapted design of controls in time-critical applications.

In view of the emerging better understanding of control complexity, a rigorous understanding of two crucial issues needs to be developed - interrelation between minimum control dimension and (effective) system dimension $[120,128]$ and the question of traps in the quantum control landscape. Even if many quantum control 
problems have no traps, i.e. their control landscapes are well-suited to the local numerical optimization approaches, this is not always the case. One then needs to resort to global optimization techniques that are numerically expensive and generally do not perform very well. Whether and how global optimization methods, such as dynamic programming, may be (better) adapted to quantum control is also an open question. The efforts to solve more complicated control problems via geometric optimal control techniques must continue with a special emphasis on open quantum systems and on non-linear dynamics [159].

Another fundamental open question is whether it is possible to connect the Lewis-Riesenfeld invariants, used for the shortcuts to adiabaticity, with the formal framework of the Pontryagin Maximum Principle of optimal control theory. This would hold the promise of combining the best features of both methods.

\subsection{Quantum feedback control theory}

\subsubsection{State of the art}

In contrast to the open-loop techniques discussed in Section 2.2, closed-loop strategies [19] are capable of coping with unpredictable disturbances. This approach is widely used in classical control theory, where information from the state of the system is fed back to the controller to correct the field in action. However, the perturbing effect of quantum measurements precludes direct application of classical concepts. It required development of new techniques tailored to quantum dynamics [15,160]. An indirect application is found in experimental closed-loop quantum control, where each cycle of the loop is realized experimentally, including preparation of a new sample in each cycle [161]. Various learning algorithms, such as genetic algorithms, have been developed to design the new control field after each iteration [17].

Control of a quantum system by feedback in a closed loop can be realized in two different ways [162] - using the measurement-based feedback approach [163] or the coherent feedback method [164]. As in classical control theory, the first option is based on a measurement process and real-time manipulation of the system depending on the measurement result. The measurement feedback approach, built on quantum filtering techniques [156], is at present mathematically well researched $[15,165]$. Quantum filtering consists in generating an estimate of the state of the system from the measurements performed on it. Measurement-based feedback has been shown to be efficient $[166,167]$, making real-time control of quantum systems experimentally feasible $[168,169]$.

In the second option, no measurement is used and the quantum system is directly connected to a quantum controller. Tests of experimental feasibility of coherent feedback control have recently led to impressive results $[170-173]$.

\subsubsection{Mid-term prospects: goals and challenges}

It is the specific quantum nature of systems that makes them susceptible to measurement back-action. Thus, in the quantum domain, finding an experiment-class adapted balance between open-loop control techniques and closedloop feedback counterparts is one of the major challenges. Because measurement-based feedback is restricted to the processing time of the classical components, which have the slowest time scale, exploiting the limits of coherent feedback control is useful as a way of building further generations of quantum technologies. An advantage of coherent feedback over its measurement-based counterpart is the reduced noise produced by the control process since there is no disturbance from a system measurement. Feedback control methods promise particular robustness and flexibility, but some important questions related to the quantum nature of the dynamics remains to be solved. In particular, there is no general theory showing that quantum controllers perform better than their classical counterparts, and if so, in which cases and under which experimental conditions.

Many other questions are still open in quantum feedback control. They range from general theories about the role of weak measurements in the control of quantum systems to feedback control of non-Markovian dynamics and the influence of model uncertainties on the feedback control. Similarly, another interesting perspective is to explore whether programmable quantum processors $[174,175]$ themselves could be used or programmed to steer quantum systems.

Most of the developments of quantum feedback control have been made in the context of quantum optics $[15,163,168,176]$. Recent technological progress has enabled advances in superconducting circuits both theoretically [177] and experimentally [178,179]. A problem that is starting to attract attention is feedback control in quantum transport [180]. In summary, the objective is to understand how the current quantum control techniques developed for quantum optical systems can be applied to hybrid systems involving quantum dots, superconducting qubits and opto-mechanical resonators. The existing quantum feedback theory has to be adapted to these new types of dynamics.

\subsection{Long-term vision}

Quantum optimal control theory has reached a level of maturity that enables tackling the question of quantum control in a world that is never fully quantum, be it due to residual couplings to the environment or due to imperfections in the controls or system characterization. The ultimate goal is to develop a rigorous understanding of the fundamental limits, as well as the opportunities for quantum control, under real-life conditions, in terms of both controllability and control design. This includes in particular control of quantum systems undergoing nonMarkovian evolution, as found in condensed phase physics and chemistry. It will most likely require a combination of 
the techniques described above and a synergy of efforts, ranging from pure mathematics through algorithm design all the way to developing techniques for integrating theory and experiment. Meeting these challenges would provide the mathematical and algorithmic underpinnings for the application of quantum control in all of the three fields described below.

\section{Atomic, molecular, and chemical physics}

\subsection{State of the art}

Within the realm of atomic, molecular and chemical physics, quantum control was first discussed in the context of chemical reactions. It was termed 'coherent control' at the time and conceived as a way to control the outcome of a reaction using laser fields [181-183]. The basic idea was to employ interference of matter waves to enhance the desired outcome and suppress all others [184,185]. A way to create the desired matter wave interference is by tailoring laser fields [181-183]. A reaction is viewed as the following sequence of events - approach of the reactants; formation of a new chemical bond; intermediate dynamics of the generated molecular complex; stabilization into the target products, typically involving the breaking of another chemical bond. Each of these steps can in principle be controlled.

The advent of femtosecond lasers and pulse shaping technology [186,187] in the 1990s allowed for experimentally testing the idea of controlling chemical reactions. In the lab, the laser pulse shape can most easily be determined in feedback loops combined with e.g. genetic algorithms [161]. Very soon after the theoretical proposals several pioneering experimental papers were published describing control of unimolecular dissociation or fragmentation, see [188-196] and references therein. Deciphering the control mechanisms underlying the optimized pulses is a complex task, and much still remains to be understood. A particularly contested question concerns the extent to which quantum coherence is involved [197-200], and at the moment, a striking example that relies on coherent quantum interference is missing.

Control strategies include both weak- and strong-field scenarios. In the first case, a wavepacket is launched, and its ensuing (ro-)vibrational dynamics is exploited. In the strong-field regime, the laser pulse coherently controls the dynamics during the pulse while utilizing the effective modification of the energy levels of atoms [201-204] or, respectively, the potential energy landscape experienced by the molecules, via the dynamic Stark effect [205-207].

Weak field control of non-resonant excitation may employ optical interferences to control e.g. the population in the final state [208]. Weak field here is defined as low order perturbation theory being applicable. It was proven early in the development of quantum control, that for weak fields in an isolated quantum system, phase-only control is impossible for an objective which commutes with the drift Hamiltonian [209]. A qualitative explanation is that under such conditions there are no interfering pathways leading from the initial to the final stationary states. Experimental evidence has challenged this assertion, claiming demonstration of weak-field phase-only control for an excited state branching ratio [210]. The phenomena were attributed to the influence of the environment. A subsequent study showed that such controllability is solvent dependent [211]. A theoretical demonstration that phaseonly control is possible in weak field for an open quantum system soon followed [212]. It is still an open question if weak-field phase-only control is possible for targets that commute with the Hamiltonian in open quantum systems. For example, can population transfer from a ground to the excited surface be phase-controlled for a dye molecule in solution? While this has theoretically been shown to be impossible if the time evolution is Markovian [213], most solvents lead to non-Markovian system dynamics.

In the context of coherent control of a chemical reaction, the bimolecular process of bond formation using femtosecond lasers remained more elusive [214-221] than bond breaking. Its coherent control was demonstrated only very recently [222]. Full control of a binary reaction - from its entrance channel of scattering reactants to the targeted products in a selected internal state - is still an open goal. Realizing this dream would create a new type of photochemistry with selective control of yields and branching ratios.

Coherent control of bond formation in the gas phase turned out to be so difficult because it starts from an incoherent thermal ensemble. The laser pulse then needs to pick those scattering pairs which show some correlation in their translational or rotational motion. Averaging over rotations can be avoided by orienting or aligning the molecules using strong laser fields [223]. Adiabatic alignment occurs in-field and is achieved with nanosecond pulses. In contrast, femtosecond laser pulses create nonadiabatic alignment that persists after the pulse is over. This second option, where the alignment is produced in field-free conditions, is more interesting in view of the applications because the aligning laser pulse does not disturb the molecular system [224,225].

Spatial averaging in the gas phase implies an integral over the beam profile and blurs coherent effects as the atoms or molecules are exposed to different intensities. Spatial resolution is thus a prerequisite for control [226]. Subwavelength dynamic localization of the laser intensity can be achieved on the nanometre scale [227], lifting the restrictions of conventional optics. Optimal control theory can be used for spatial shaping of the laser fields used in nanoexcitation [228].

Another way to overcome thermal and spatial averaging is by cooling and trapping the atoms or molecules. Coherent control was suggested as a method to cool internal molecular degrees of freedom $[112,229]$. This has been realized experimentally for cooling vibrations of ultracold cesium dimers [230] and rotations of trapped aluminum hydride ions [231]. While restrictions imposed on the cooling efficiency by the molecular structure can be circumvented using optimal control [232], a persistent challenge to cooling internal molecular degrees of freedom are the 
timescale separation between vibrations and rotations as well as the enormous bandwidths that are required for strong bonds. In order to prepare molecules that are cold in their translational degrees of freedom, molecules are assembled from atoms which are much easier to cool. However, a major problem in creating molecules from atoms is the extreme change in time and length scale. Optimal control was studied to overcome this issue [233]. Initial experiments employed the simple scheme of a chirped pulse to compress two atoms to closer proximity [234]. This can be viewed as a first step in ultracold laser-induced, i.e., photoassociation. Association yielding ultracold molecules in a single internal quantum state has been demonstrated employing magnetic field ramps (magneto-association) followed by STIRAP-type protocols [235,236]. An experimental challenge still unfulfilled is complete coherent control of ultracold photoassociation.

One of the important issues here, particularly in polyatomics, is the need for accurate ground and excited potential energy surfaces to design optimal pulses a priori, as well as to interpret the mechanism of pulses that are found by experimental optimization. For this reason there has been significant interest in recent years in inverting the information in optical spectroscopies in order to reconstruct excited state potential energy surfaces [237-239].

In addition to making or breaking chemical bonds, coherent control has demonstrated its versatility in studying energy transfer [240], light harvesting [241,242], and spectroscopy. The latter includes in particular non-linear and multi-dimensional spectroscopies [243-246] and microscopy [243,247,248]. In this context, coherent control allows to increase both the resolution and the specificity to a single molecule. Originally developed with phaselocked time-delayed laser pulses, non-linear and multidimensional spectroscopies utilize the interference created by these pulses. Laser pulse shaping not only lifts the constraint of having separate pulse beams in non-linear spectroscopy and microscopy [243-245], it also addresses naturally the requirement to decipher the interference which is at the basis of the non-linear spectroscopies. Eventually, this understanding has lead to the use of laser pulse shaping in optical imaging with compensation of the effect of scattering media such as biological tissue [249,250]; optical microscopy with enhanced chemical sensitivity and contrast [251-254]; chemical analysis and detection via optical discrimination [255-258]; cancer diagnosis [259]; and material processing [260]. Similarly, laser pulse shaping is expected to enhance chemical sensitivity in other detection methods such as mass spectroscopy [261,262].

Initially, the coherent control of molecules, be it in the context of chemical reactions or non-linear spectroscopies or energy transfer, considered the dynamics of the nuclear degrees of freedom, using femtosecond laser pulses as the main workhorse. More recently, the focus has shifted to controlling electron dynamics. This is being made possible by the development of advanced X-ray sources which probe the dynamics of electrons within atoms and molecules on attosecond time scales. Their potential for exploring the quantum nature of the nanoworld is unprecedented. For example, using XUV and X-ray light for multidimensional spectroscopy could probe valence excitations locally on different atomic sites in a molecule. This would be invaluable for understanding energy transfer in biological systems and quantum devices. The use of $\mathrm{X}$-ray light sources is currently facing a number of challenges that can be tackled by quantum optimal control. First of all, the large energy of XUV and X-ray pulses results in a high probability of ionization, reflecting the problem of controllability when a continuum of states is involved. This has been addressed in a recent study, where optimal control theory was used to predict experimentally feasible pulses to drive XUV-Raman excitations through the ionization continuum [263].

Another control problem is the creation of the XUV light pulses themselves. In particular, high harmonic generation, where a very strong near-infrared femtosecond laser pulse accelerates an electron in such a way that it emits XUV light, is an ideal candidate for coherent control: theoretical predictions for optimum driving should be easy to adapt in experiment, given the existing pulse shaping capabilities. The challenge that high harmonic generation poses to optimal control theory is a frequency-domain target $[103,264,265]$. A conclusive answer whether shaping the femtosecond laser pulses can improve high harmonic generation has not yet been provided.

Optimal control theory was first applied to chemical reactions using Krotov's method [266,267] and gradient ascent $[268,269]$. The theory was quickly extended to Liouville space $[112,270]$ to treat condensed phase situations and cooling. Optimal control techniques have also been applied with success to molecular alignment and orientation in gas phase [223]. The design of optimal solutions has allowed to reach the best possible degree of alignment and orientation [271] under experimental constraints such as temperature [272] or collisions [273]. In addition to improving existing control strategies, optimal control has also been used to explore new regimes of alignment dynamics such as planar alignment [274].

The major experimental constraint in experiments with shaped femtosecond laser pulses is the fixed bandwidth. This can be accounted for in optimal control theory by including frequency filtering $[105,111,275,276]$ or by imposing spectral constraints $[109,277]$. The effect of additional constraints is to pick a different solution out of the many solutions that typically exist in quantum optimal control $[134,135,277]$. Then a natural follow-up task to any optimization is to determine the control mechanism encoded in the optimized pulse. To date, a systematic approach tackling the control analysis is still lacking.

\subsection{Mid-term prospects: goals and challenges}

Thirty years after the conception of reaction control, it is fair to ask whether the idea of coherently controlling a chemical reaction can work at all. In this respect it is important to realize that the basic ingredients for achieving this goal have all been developed. The challenge that remains to be overcome is their assembly and application 
to a specific reaction. The dream of coherently controlling a chemical reaction all the way from its entrance channel to the reaction products thus seems to be within reach. It includes the controlled formation (or photoassociation) of a new chemical bond, the controlled dynamics of the intermediate complex, most likely involving a conical intersection, the controlled cleavage of another chemical bond as well as the stabilization of the reaction product. Realistically, demonstration of control over a complete reaction can be expected within the next few years for a sufficiently simple reaction complex, involving only a few atoms.

Experimental techniques are currently developed to trap and cool a single molecule. These are particularly advanced for single molecular ions [278,279]. The molecules can be simple diatomics or large biological chromophors such as rhodopsin. Such trapped species are ideal candidates for control, inducing for example isomerization in rhodopsin. Instead of trapping and cooling molecules directly, they can also be assembled from cold atoms one by one. The number of different atoms which have been cooled and trapped has been increasing steadily. Atoms other than the first row alkali metals have the potential for complex chemistry with multipole bonds. This raises the challenge of assembling molecules from these constituents. The experimental obstacle is the development of pulse shaping techniques in the picosecond and nanosecond range.

Another mid-term goal for quantum control is the control of electron dynamics. The ability to control electrons implies Angstrom-scale ultrafast imaging methods which can be realized in the form of laser-induced electron diffraction and high-harmonic spectroscopy. Specific mid-term goals that appear to be within reach using these tools include the control of subfemtosecond charge migration; the controlled generation of spin-polarized electrons from laser ionization; recognition of the absolute configuration of chiral molecules with shaped laser pulses; and, using high-harmonic spectroscopy of molecules, ultrafast imaging of structure and dynamics on sub-atomic length scales.

\subsection{Long-term vision}

\subsubsection{Synthesis}

The ultimate chemical synthetic challenge is to assemble large chiral molecules from elementary building blocks. Currently such syntheses are carried out in solution where the chemical products are stabilized by entropy generation caused by heat transfer to the environment. The vision is for synthesis by photoassociation via polarization shaped light where the final product is stablized by laser cooling and trapped by light.

\subsubsection{Analysis}

The vision is for a light field tailored to a specific molecule or functional group generating a specific physical out- come such as light emission or ionization. Such a capability will enhance the threshold of detection of specific molecules, e.g. in hazard detection or in medical applications. Combined with spatial super-resolution, the resulting analytic methods are also expected to find applications in molecular-scale microscopy.

\section{Magnetic resonance}

The optimal control of spin dynamics is at the heart of well established magnetic resonance technologies and of emerging new fields of quantum technologies. Nuclear magnetic resonance spectroscopy (NMR) [280-282], electron spin resonance spectroscopy (ESR) [283] and magnetic resonance imaging (MRI) [284] are based on the control of nuclear or electron spins with the help of time-dependent electromagnetic fields. Magnetic resonance is one of the most impressive success stories of quantum control and technology.

The mathematical description of the state and dynamics of nuclear spins or electron spins is essentially identical to the canonical description of abstract quantum bits (qubits). In terms of theoretical and experimental control of spins or qubits, NMR had a long head start compared to other quantum technologies because the NMR community has actively explored and developed spin system control methods for more than 60 years. This was driven by many specific and practically important applications in physics, chemistry, biochemistry, biology and medicine. The interdisciplinary impact of quantum-control enabled magnetic resonance is impressively reflected by Nobel prizes in physics (Felix Bloch, Edward Purcell, 1952), chemistry (Richard Ernst, 1991; Kurt Wüthrich; 2002), and medicine (Paul Lauterbur, Peter Mansfield, 2003) [285-287].

Today, NMR is arguably the most important tool in chemistry to determine molecular structure and dynamics of molecules. ESR is an essential technique in radical reaction chemistry, catalysis, electrochemistry and photosynthesis research. MRI is one of the most informative and frequently used modalities in medical diagnostics. The huge range of practical applications has generated a multi-billion dollar branch of the instrument manufacturing industry (Bruker, Siemens, Phillips, General Electric, JEOL, etc.). This in turn has resulted in the continuous development of more and more sophisticated instruments with superb flexibility in terms of the available control schemes: for example, arbitrary waveform generators and linear amplifies have been standard NMR and MRI equipment for more than three decades (and have more recently also become available with sub-nanosecond time resolution for ESR applications [108,288]). With their help, even very complex pulse shapes can routinely be implemented with high fidelity [289]. The excellent agreement between theory and experiments (as a result of the highly accurate theoretical description of the physics of coupled spins and the availability of very reliable hardware to implement virtually arbitrary pulse sequences and shapes) also has made NMR an attractive testing ground for the experimental demonstration of new control approaches for 
finite-dimensional quantum systems in general. Concepts of quantum control and sophisticated quantum-control design tools developed in the field of NMR have found many applications in other fields, such as in quantum information processing [290-292], optics (photon echos) [293], in neutron scattering and in the control of nano devices based on quantum dots, artificial atoms, etc.

Two important emerging fields of magnetic resonance are hyperpolarization methods for bulk NMR and the control and measurement of individual spins or spin systems, e.g. of NV centers in diamond, with many potential applications in sensing and quantum information processing [294-296]. Hyperpolarization techniques (also known as spin cooling), [297-299] can generate highly polarized non-thermal spin states. Hence, the relatively low sensitivity of NMR (due to the small magnetic moments of nuclear spins and the resulting weak thermal polarization) can be overcome by using a variety of approaches. In particular, there are two different methods that have recently become increasingly popular in practical applications. The first one is based on transfer of the much higher thermal polarization from unpaired electrons onto the nuclear spin ensemble in a process called dynamic nuclear polarization (DNP) [300-302]. The second method involves the use of parahydrogen and a transfer of its highly populated singlet spin state onto nuclear spins in receptor molecules [303]. Both methods have been known for many years, but only recently significant progress has been made in terms of a full quantum description of the underpinning spin physics and the optimization of the required experimental hardware.

The detection and control of individual nuclear spins close to nitrogen-vacancy (NV) centers in diamond [304,305] is an excellent example of a new area of optimal control of individual spin systems. In these experiments, single nuclear-spin detection is achieved by an efficient readout based on couplings of the nuclear spins to electron spin states and their detection using optical techniques. In the field of spintronics, spins of individual atoms (such as ${ }^{31} \mathrm{P}$ donors) or quantum dots can be electrically detected and controlled by radio- or microwave pulses with high fidelity [306,307].

\subsection{State of the art}

The design of pulse sequences that provide maximum sensitivity, selectivity, as well as maximum resilience to instrumental imperfections, is central in the ongoing effort to improve magnetic resonance technologies. Both analytical and numerical methods have been used to design multiple-pulse sequences (including composite or shaped pulses).

Since the 1950s, the field of NMR has been a highly active "breeder" for methods to control finite-dimensional quantum systems. The wide spectrum of applications has motivated the development of more and more sophisticated tools for quantum control in a quest to push the experimental performance to their limits. Pulsed magnetic resonance techniques such as the two-pulse Hahn echo and the three-pulse stimulated echo [308] were important milestones on the path to multiple-pulse sequences, which often consist of tens or hundred thousands of individual pulses with defined amplitudes and phases. This was enabled by the development of theoretical tools such as average Hamiltonian theory (AHT) [309] and the symmetry analysis of composite pulses and multiple-pulse cycles and supercycles [282,310-312].

More recently, sophisticated theoretical approaches of geometric optimal control theory $[4,12,68,69,71,82,89]$ have been applied to quantum control problems motivated by magnetic resonance applications. Although these analytical approaches are typically limited to low-dimensional quantum systems, they are able to provide the best possible solutions to a given problem by proving global optimality. The resulting physical performance limits are important theoretical results in their own right and also provide benchmarks for numerical and experimental optimization techniques. Geometric optimal control has been very successfully applied to highly non-trivial examples of uncoupled and coupled spins both in the absence and presence of relaxation (dissipation and decoherence). For example, based on geometrical optimal control analysis, the minimal time for quantum gates in systems of two and three coupled spins have been determined and experimentally implemented $[71,89,313]$. And in this context, the socalled quantum-gate design metric was discovered, which plays an important role for the design of so-called geodesic unitary gates $[71,314]$. For example, time-optimal control schemes for the simulation of effective trilinear couplings in systems with only next-neighbor couplings were derived based on geometric optimal control and experimentally demonstrated using NMR. This is a particularly impressive example, because in the limit of small time increments $\Delta t_{\text {sim }}$ under the simulated trilinear Hamiltonian, the actual duration of the controls also approaches zero, whereas previous approaches based on AHT have a minimal duration of $1 /(2 J)$, i.e. for $\Delta t_{\text {sim }} \rightarrow 0$, the gain of the optimal-control schemes approaches infinity [71,314]. Examples of geometric optimal control applications involving singular arcs [68] in NMR are relaxation-optimized polarization transfer experiments $[78,79,82,315]$, minimal-time controls for the saturation of spins [77], for maximizing contrast in MRI [88] and for maximizing the achievable signal-to-noise ratio per unit time [316,317].

Since the 1980s, numerical algorithms such as conjugate gradients, downhill simplex (Nelder Mead), genetic algorithms and simulated annealing have been applied to find efficient and robust quantum-control schemes for magnetic resonance applications [318-323]. Powerful optimal-control algorithms based on the Pontryagin Maximum Principle (PMP) were applied already since the mid 1980s to the design of shaped pulses for the manipulation of nuclear spin ensembles [324-326]. Potent variants of these PMP-based algorithms were also developed in the context of NMR applications for coupled spins in NMR spectroscopy and quantum information processing $[92,93,327]$. They are able to optimize pulse sequences for an ensemble of spin systems with 
realistic parameter ranges for detunings, scalings of the control field etc., i.e. they can take into account variations or uncertainties of experimental parameters. Numerical ensemble-control methods have resulted in extremely powerful control schemes with unprecedented resilience to pulse imperfections, while at the same time taking experimentally bounded control amplitudes and pulse energy limits into account.

In magnetic resonance, an efficient and user-friendly software landscape is starting to emerge - there are a few software packages that support sophisticated quantum mechanical magnetic resonance simulations as well as optimal control. In particular, SPINACH [101] supports all forms of magnetic resonance spectroscopy under the same roof, implements sophisticated spin relaxation theories as well as most mainstream optimal control algorithms that are presently used in magnetic resonance spectroscopy: GRAPE [92], GRAPE-BFGS [93,94], Krotov [96] and Krotov-BFGS [99]. Another advantage of SPINACH is the availability of polynomially scaling spin dynamics simulation algorithms that make previously intractable NMR and EPR simulation (and therefore control) problems accessible $[121,328]$. In the solidstate NMR community, the SIMPSON software package (SIMulation Program for SOlid state Nuclear magnetic resonance) has been the most extensively used generalpurpose software. It also includes an optimal control toolbox to facilitate robust experiment design [100]. MATPULSE [329,330], DYNAMO [94] and QuTiP [102] are versatile Matlab and Python based simulation and optimization programs. Other software packages for the simulation and/or optimization of spin dynamics include SIMONE [331], OCTOPUS(SI) [332,333], Gamma [334] and SPINEVOLUTION [335].

Both analytical and numerical methods have proven very effective for two- and three-level systems $[72,74-77,80,159,336-348]$, two uncoupled spins [349,350], and two coupled spins [83,85,89,351-355]. Significant progress has been made in understanding of how to optimally control coupled spin systems with more than two spins $[65,71,86,87,313-315,356-374]$. Recent advances include robust broadband and band-selective pulses in NMR and ESR. Depending on their role in a given experiment, universal rotation (UR) pulses (e.g. for refocussing) or point-to-point (PP) pulses (e.g. for excitation or inversion of spins) are required. Systematic studies of the offset bandwidth (range of detunings) and robustness with respect to scaling of the control amplitude (width of $B_{1}$ inhomogeneity distribution) of optimized PP $[52,53]$ and UR pulses have been performed [55] for experiments where the maximum control amplitudes (e.g. in many applications of NMR or ESR spectroscopy) or the pulse energy (e.g. in MRI) are experimentally limiting factors. For a desired fidelity, the bandwidth covered by a (composite or shaped) pulse can be made much larger than the maximum control amplitude (maximum Rabi frequency) at the price of increased pulse duration. Surprisingly, it was found empirically that the pulse duration typically scales only linearly with the desired offset range of operation. Hence, unprecedented fidelities can be achieved that are e.g. required for quantitative NMR applications or for quantum error correction schemes. In addition, it was demonstrated that coherence transfer elements can be designed that are not only resilient to offset and control amplitude variations but also to variations in coupling constants $[355,375]$.

In general, UR pulses are significantly longer than PP pulses for the same error resilience, which was exploited in a new strategy to build complete sequences based on standardized UR pulses when necessary and standardized PP pulses whenever they are sufficient [376]. The recent application of optimal control methods to the problem of heteronuclear decoupling yields not only significantly improved performance $[377,378]$ but also unprecedented flexibility in the design of tailored decoupling sequences [379-381]. Individual pulses were also optimized for Carr-Purcell-Meiboom-Gill echo train sequences [382,383]. Beyond individually optimized pulses, the simultaneous optimization of pulses provides significant performance gains by exploiting cooperative effects either in a single scan [384] or in multiple scans [385] with first applications in Ramsey-type experiments and in Hahn echo sequences.

In liquid-state NMR, optimized broadband pulses have found applications in a number of experiments. Early examples are the CLIP-HSQC [386] and P.E.HSQC [387] for the measurement of one-bond and geminal couplings as well as more recent experiments involving homonuclear decoupling [388] and the rapid acquisition of heteronuclear correlation experiments [389]. All these experiments are of special interest as they are recorded in large numbers every day in chemical laboratories and improvements in robustness, accuracy, or acquisition time are of high interest.

For the specific conditions of solid-state NMR and experiments in oriented systems, specific pulse sequences have been optimized [390-396]. In ESR spectroscopy, the first broadband optimal-control pulses have recently been developed and experimentally implemented [108,288]. In this context, the efficiency of novel approaches to take into account transient effects due to transfer line effects and limited resonator bandwidth was both numerically and experimentally demonstrated [108].

Motivated by magnetic resonance imaging applications, optimal control pulses for MRI have been developed [88,324-326,330,397-400]. Applications include improved spatially selective excitation schemes [106,401,402], pulses with minimal radio-frequency $(\mathrm{RF})$ power and pulses that counteract RF inhomogeneity in parallel transmission at ultra-high field [330,398]. For chemical exchange saturation transfer (CEST) imaging, chemical exchange effects were taken into account in pulse sequence optimizaion [403].

For DNP, optimal polarization transfer schemes from electron to nuclear spins have been investigated for a small set of relatively simple model systems [302,404-407]. Optimal control was also used in conjunction with the use of parahydrogen to generate high nuclear spin polarization. 
In particular, it was shown that the initial longitudinal two-spin order arising from the hydrogenation reaction can be equally distributed between several spins and converted into detectable magnetization [408].

Geometric and numerical tools from optimal control theory have not only provided pulse sequences of unprecedented quality and capabilities, but also new analytical insight and a deeper understanding of the mode of action of optimal pulses. Numerically optimized pulses can often be interpreted as robust implementations of analytically derived optimal trajectories [77]. These can be understood based on geometrical concepts [341]. In addition, useful tools have been developed to analyze complex pulses [409] and the resulting dynamics in coupled spin systems $[405,410,411]$.

\subsection{Mid-term prospects: goals and challenges}

An important goal is to make optimal control algorithms easier to use, generally applicable and to further increase their speed. Depending on the applications, very different convergence rates are encountered and a systematic characterization of optimal control landscapes is still missing. With more efficient numerical optimizations methods, optimal control theory will make it possible to design problem-, sample- and patient-specific pulse sequences on the fly. The fast reoptimization of pulse sequences or of sequence elements, e.g. in response to the presence of magnetic susceptibility jumps, will significantly improve their performance. In addition to MRI applications, this could be important in production or process monitoring by NMR, where the pulse sequence should be able to adapt to the sample in the same way as shim currents currently do - examples are magnetic susceptibility and tuning variations in imaging, metabolomics and oil well logging [382].

Optimal control methods are expected to reduce the time that is required to determine structural and dynamical information on biomolecules (e.g. proteins). In this area, coping with large coupled spin networks, especially in the presence of relaxation, is computationally hard and further improved numerical/analytical approaches are highly desirable. Characterizing the experimental imperfections, fine-tuning the spectrometer and setting up the experiment is time-consuming and efficient closed loop feedback-based automatic procedures have to be developed, implemented and integrated with the adaptive design of pulse sequences to automate this process.

In the field of medical imaging it is expected that optimal control methods will lead to more sensitive and more efficient pulse sequences, such that a patient has to spend less time in a scanner for an examination. This may be achievable by using multi-band excitation techniques and optimized image acquisition based on multiple transmit and receive channels. Optimal control methods are also expected to help in the extension of the clinical applicability of ultra-high-field scanners and to provide in-vivo spectroscopy with improved diagnostic value. Apart from better localized excitation, progress in the field of magnetization preparation (e.g. reducing the $\mathrm{B}_{0}$ sensitivity of fat saturation) might be achievable. Improved saturation pulses are expected to be useful for many different tasks, from chemical shift imaging (CSI) and chemical exchange saturation transfer (CEST) imaging [403] to single voxel spectroscopy. Also methods for improved quantification accuracy and biomarker imaging are highly desirable.

Small molecule NMR spectroscopy forms the basis of chemical analysis on a molecular level in solution. Applications are already manifold, but are expected to increase significantly during the next decade. Identification and quantification of compounds in complex mixtures is needed. Especially for quantification tasks, methods derived from optimal control will deliver more reliable and robust results. Optimal control sequences are likely to improve the detection level of side products, making chemical products safer and better defined. Quality control of food, pharmaceuticals and other products as well as metabolomics-type applications and personalized medicine will benefit from improved NMR.

In the field of hyperpolarization, further hardware advances are expected, in particular in generating and modulating high-frequency microwave fields and it is envisaged that optimal control methods will play an important role in the development of more sophisticated experimental schemes to transfer the electron polarization to surrounding nuclear spins. Ideas on how to control electron-nuclear spin systems in a optimal way have already been published and discussed. This includes the manipulation of the nuclear spins using the anisotropy of the hyperfine interaction $[412,413]$ and the exploitation of repeated generation of dipolar spin order to enhance polarization transport by spin diffusion [414]. Since more sophisticated catalysts are being developed for parahydrogenation reactions [298], it is also anticipated that pulse shapes derived from optimal control principles will be more frequently used to maximize the achievable polarization and to mediate polarization transfer to specific atoms.

\subsection{Long-term vision}

Important theoretical and practical aspects of optimal control in magnetic resonance are the physical limits of quantum dynamics. On the one hand, the questions of quantum state reachability in dissipative systems remain largely unresolved. On the other, many practical usage scenarios are time-constrained, and a more general understanding of the best possible performance in a give amount of time is highly desirable.

A very desirable outcome of the continued progress in optimal control technology could be that, for a given level of performance, the use of optimal control sequences could significantly reduce the instrument costs as well as costs of sample preparation and purification. Sophisticated shim coils, frequency locks, complicated combinations of susceptibility matched materials in NMR probes and other expensive arrangements had originally been introduced to maximize spectral resolution and selectivity. If both could be achieved by tailored pulse sequences under less than ideal conditions, the complexity could be transferred 
from the instrument design to the mathematical optimization procedure. The concomitant reduction in hardware cost could result in more affordable instruments, e.g. for MRI examinations. Also, the integration of control design with image reconstruction and spectral calculation in NMR and with instrument design could result in better performance. The combination of open loop and feedback strategies may result in fast and fully automated tuneup procedures, which would further reduce the required experimental time.

A long-term vision for magnetic resonance techniques is the detection of the nuclear spins of individual molecules, such as a protein. The ability to image the shape of a single molecule similar to the way we can image humans today would revolutionize structural biology and pharmacology. The recent developments in sensing using NV centers in diamonds (see Sect. 5) might eventually reach this goal, as well as generate completely new application areas, e.g. in medical diagnostics.

\section{Quantum information and communication}

\subsection{State of the art}

Quantum technologies (see, e.g., [2]) exploit quantum coherence and entanglement as essential elements of quantum physics. Applications include high-precision measurements and sensing, which would reach unprecedented sensitivity, the simulation of physical and biological systems, which would be impossible to study otherwise, and quantum information processing, which would allow to solve computationally hard problems. Successful implementation of quantum technologies faces the challenge to preserve the relevant nonclassical features at the level of device operation. More specifically, each task of the device operation needs to be carried out with sufficient accuracy, despite imperfections and potentially detrimental effects of the surroundings. Quantum optimal control not only provides toolboxes that allow for identifying the performance limits for a given device implementation, it also provides the protocols for realizing device operation within those limits.

Prominent tasks include the preparation of useful quantum states as well as implementation of quantum operations. The power of the quantum optimal control approach for implementing these tasks has very recently been demonstrated in a number of impressive experiments. For example, nonclassical motional states of a Bose-Einstein condensate were prepared with optimized control sequences for wavepacket interferometry [415], and the loading of an ultracold atomic gas into an optical lattice was improved [416]. With respect to quantum operations, quantum optimal control allowed for error resistant single-qubit gates with trapped ions [417] and for single qubit gates without the need for invoking the rotating wave approximation in nitrogen vacancy (NV) centers in diamond [418]. For the latter platform, optimal control is also at the basis of a spectroscopy protocol allowing to image nanoscale magnetic fields [295]. In quantum processor candidates based on superconducting circuits, leakage to non-computational states in the most common type of qubit, the transmon [419-421], was avoided and frequency crowding was accomodated $[422,423]$ thanks to optimal control results. Closed-loop optimal control [150,424] enabled fine-tuning of gates that were determined manually, allowing them to reach consistent record fidelities within this platform. Control of donor qubits in Si has been achieved and characterized [425]. Control strategies for spins in semiconductors currently trade off conceptually simple single-spin schemes $[426,427]$ with more robust and accessible two- and three spin techniques [428-430]. The short natural time scales suggest adiabatic schemes to be attractive [431-433] In view of scaling up control, the design and implementation of unitary maps have recently been demonstrated in a 16-dimensional Hilbert space, spanned by the electron and nuclear spins of individual cesium atoms [434].

The use of control methods in a broader sense has allowed for further significant experimental achievements, such as to improve of the coherence of a qubit, realized by the electron spin in an NV center, using dynamical decoupling [435]. A famous further example of high-end control techniques is the Paris experiment of stabilizing a quantum state with predefined photon number via real-time closed-loop feedback [168], which required to include the noise back-action of controls onto the system by way of stochastic differential calculus.

These experimental achievements were preceded by a large number of theoretical predictions on how optimal control may improve or enable quantum state preparation, operation and readout. State preparation protocols include transport of atoms [436] and ions [437,438] as well as transport in a spin chain $[86,356,359,363,369,439,440]$, photon storage [441], preparation of squeezed states [442], cluster-states [443], non-classical states in a cavity [444] or in spin chains [364,365], as well as preparation of a quantum register [122] and many-body entangled states $[123,445,446]$ - to name just a few.

Likewise, optimal control helped to implement highfidelity quantum gates such as two-qubit gates with neutral atoms in dipole traps [447,448], on atom chips [449], or with Rydberg atoms $[450,451]$, two-qubit gates between ions [452], between an ion and an atom [453], error-correcting qubit gates of electron and nuclear spins within single NV centers [454], or entangling gates between distant NV centers [296]. For superconducting qubits [455], two-qubit gates were optimized, starting from Cooper pair boxes [456-459] to modern transmonbased schemes $[117,460]$. In these optimizations, special attention was paid to robustness against noise $[456,461]$ which can even be used as a tool for control [60]. Also, readout has been addressed $[462,463]$. In order to adapt to the strong filtering of control lines in superconducting qubits, transfer functions had to be taken into account $[107,108,110,464]$ and experimental fluctuations and noise were accomodated $[117,364,465]$. Fidelity limits on two-qubit gates due to decoherence were studied for Markovian [56,114,117] as well as non-Markovian [116] time evolutions. It is noteworthy in this context that 
non-Markovian time evolutions also play a role in quantum simulation, e.g., of collision models [466].

In view of quantum computation, it has been suggested how to retain universality in spite of limited local control $[65,364]$ by using environmental degrees of freedom [60]. The Jones polynomial, i.e., a central invariant in knot theory, can be evaluated, using an NMR spin ensemble at ambient temperature, in an algorithm equivalent to deterministic quantum computing with a single pure qubit [467-469].

In order to obtain these results, the quantum optimal control methodology had to be adapted to the requirements of Quantum Technologies. Optimization algorithms had to be derived for specific quantum gates [470-472] dissipative evolution as seen in the reduced system dynamics [56,113,114,461,473], or exploiting invariants in system-bath models [474], optimization up to local equivalence classes [475], which can also be used for arbitrary perfect entanglers $[476,477]$ or optimizing for many-body entanglement [478]. Moreover, control techniques were adapted to non-linear dynamics as found in a BEC [479-481] and to general dynamics, functionals and couplings to be controlled [98]. Manybody systems can be optimized numerically with the chopped random basis (CRAB) method [122,123] by interfacing the time-dependent density matrix renormalization group (t-DMRG) with parameter optimization via e.g. the simplex algorithm. Other techniques specifically cover robustness against experimental fluctuations or noise $[56,117,364,444]$ or filters in experimental implementation of controls $[107,108,110,464]$.

\subsection{Mid-term prospects: goals and challenges}

The field of quantum technologies has matured to the point that quantum enhancement is explored beyond quantum computation only. Devices such as quantum simulators or quantum sensors are currently under active development. Control methods will be crucial to operate these devices reliably and accurately. This involves the device preparation, or reset, the execution of the desired time evolution, and the readout of the result. These tasks set the agenda for the next few years.

More specifically, central mid-term milestones include the robust implementation of gates in a multi-qubit architecture, finding solutions to readout and fast reset limitations, automatization of key tasks of surface code error correction and optimal as well as robust generation of multi-particle entangled states for a variety of quantum technology platforms. All of these will require decoherence control.

A main challenge for optimal quantum control is to reach convergence of numerical optimal control and experimentation. To date, either optimal control is used for computer-aided discovery of analytical schemes that can be remodeled in an experiment or the quantum processor itself is employed to calibrate gates. In order to better combine numerical optimal control and experimentation, the modeling of qubits as well as errors and other nonidealities of the system, in particular for open systems, needs to be improved and the robustness of pulses enhanced. Also, optimized pulses should initially be reduced to few parameters before more complicated and effective solutions can be pursued. On the other hand, pulse shaping platforms need improvement.

Coming to specific quantum technology platforms, applications of quantum control in superconducting qubits should follow the current European thrust towards analog and digital quantum simulation and lead to the preparation of entangled ground states, fast and accurate quantum gates and tools for quantum machines. For some instances, compatibility with quantum error correction is desired. For trapped ions, it seems realistic to combine quantum gates with ion transport in segmented traps, using optimal control techniques, which can also be applied to the systematic optimization of pulse sequences for efficient generation of complex operations. In the field of cold atoms, challenges for optimal control are twofold. On the side of quantum simulations, the goal is to enable highfidelity preparation and manipulation of many-body quantum states of increasing complexity, with and without local control. On the side of quantum communication, the goal is to enable efficient interconversion between flying qubits and quantum memories via coherent atom-photon coupling, with and without cavities. In the case of color centers in diamond, with major applications in quantum sensing, the goal is to enhance the sensitivity of the defect spins employed as quantum probes via improved protection from environmental noise e.g. through dynamical decoupling techniques.

In general, control techniques are expected to contribute to decoupling and dissipative stateengineering [151-153], for instance in view of enhancing the lifetime of quantum memories. In order to improve the lifetime of a quantum register, control can also be used to implement error-correcting gates and circuits [454]. Moreover, while quantum compilation, i.e., the translation of a unitary gate into the machine language of pulses and evolutions, can readily be done via optimal control up to some 10 qubits [482], a scalable assembler of elementary gates (up to 10 qubits) into many qubits is an open problem that may benefit from tensor-contraction techniques.

Both numerical optimal control and closed-loop control are expected to be useful for tackling these goals. Numerical optimal control has the advantage of versatility, whereas closed-loop control can easily be tuned to specific tasks such as determining parameter uncertainties. A hybridization of both approaches is conceivable as well. The main difficulties that need to be overcome to reach the above mentioned mid-term milestones are a sufficiently accurate modelling of complex quantum dynamics to build control on top, integration of tomography and system identification with optimal control, efficient ways to take into account experimental constraints and uncertainties, and bridging the gap between the quantum control community and the communities of the respective quantum technology platforms. 


\subsection{Long-term vision}

Several current quantum technology platforms show a strong scaling potential. Thus in the long term, control schemes need to be made scalable. This represents a severe challenge, but meeting this challenge will make quantum control a basic building block of every quantum technology and ensure, at the same time, their proper functioning in a world that is only partially quantum.

Take the examples of superconducting qubits, NC centers or spins in $\mathrm{Si}$, where fabrication is a key task that could and should be improved by control techniques. The controlled adjustment of fabrication parameters should be simple, and the qubits should to a certain extent be robust to the influences of the rest of the architecture they are placed in. Independent of a specific platform, error correction at large, for instance by toric codes [483,484], is one of the strategic long-term goals that is expected to benefit from control techniques given recent advances by randomized benchmarking [424]. To this end, system-identification protocols matched with optimal control modules will be of importance. A pioneering step in this direction was made by the ADHOC technique [150] that combines open-loop control as a first step with closedloop feedback learning (with implicit parameter identification). Moreover, taking quantum control algorithms to match with tensor-contraction techniques in order to address quantum-many body systems (where first steps have been made by CRAB $[122,123]$ ) is expected to pave the way to more accurate handling of experimental quantum simulation setups.

In short, quantum control will be the means to get the most performance out of an imperfect system and combine challenging physics at the few-qubit level with engineering at the multi-qubit level. This should aim for example at enabling quantum simulations that are impossible on classical computers. In addition, the realization of the following long-term goals, using optimal control techniques, seems challenging yet conceivable: demonstrating the practical usefulness of engineered quantum states, for example in quantum metrology; implementing reliable strategies for the control of mesoscopic systems; exploring the dynamics of quantum many-body systems beyond equilibrium; and understanding the microscopic origin of thermodynamic laws. In other words, the long-term goal of quantum optimal control for quantum technologies is to develop a software layer enhancing the performance of quantum hardware for tasks in computing, simulation, communication, metrology and sensing beyond what is achievable by classical means, enabling the achievement of quantum supremacy [485].

\section{Prospects for applications and commercial exploitation}

Quantum-control enabled technologies have the potential for truly revolutionary innovation. More sophisticated control techniques would make current technologies more powerful and also help to create novel technologies, e.g. highly sensitive magnetic detectors, microscopic temperature measurement devices, molecular imaging tools etc. Better control of quantum systems has the potential to significantly reduce instrument costs, replacing large NMR spectrometers with small and portable devices with many new fields of applications.

Quantum optimal control applications broadly fall into two classes: applications to novel quantum technologies and applications to chemistry-related areas such as spin resonance. Novel optimal control strategies have already been implemented in commercial NMR spectrometers and implementations of optimal control sequences in MRI are being pursued. This trend is expected to continue. Other chemistry-related applications where coherent control plays out powerfully are imaging, optical microscopy and various variants of spectroscopy as well as chemical analysis where shaped laser pulses improve the resolution and enhance the specificity to a particular molecule. Areas of application range from remote chemical detection all the way to cancer diagnosis.

On the other end of the spectrum, there is presently an emerging industrial effort in quantum computing lead by IBM, Google, and Microsoft. The first two companies have invested into the development of superconducting qubits and both of them use optimal control techniques [424,459]. This is no coincidence - optimal control can have impact in systems that have reached some technical maturity in research laboratories, which is the point at which industry gets interested. Further industrial perspectives will be linked to further development of the quantum technologies industry. Early convergence could happen in quantum sensing, which takes sensing ideas similar to those appearing in spin resonance and combines them with ideas from quantum technologies.

\section{Conclusions}

Quantum control is a key facilitator for spectroscopy and imaging as well as AMO physics and emerging quantum technologies for computation, simulation, metrology, sensing and communication. For all these applications, it is crucial to reach the required precision given experimental limits on control amplitudes, power, timing, accuracy of instruments as well as the ever-present interaction with the environment. Optimal control theory provides a framework to identify which quantum tasks can be accomplished with what precision in the presence of decoherence and experimental imperfections and limitations.

Quantum control systems theory will require the integration of control aspects at many different levels. Future quantum technologies will rely on integrated architectures of hybrid quantum systems [486] with e.g. nuclear spins for long-term storage, quantum-nanomechanical devices for sensing and photons for the communication of quantum states. This will require also the integration of quantum mechanics in engineering education and vice versa. It will be necessary to establish strong links of quantum control 
experts to quantum engineering and to the manufacturing of quantum devices.

Due to its interdisciplinary nature with applications in many fields, future advances in the optimal control of quantum systems will require the combined effort of people with expertise in a wide range of research fields. Only the close link of basic research, development and applications will open scientifically and economically rewarding perspectives and will foster the innovation potential of emerging quantum technologies in an optimal way. The Virtual Facility for Quantum Control (VF-QC) under the umbrella of the EU Coordinated Action for Quantum Technologies in Europe (QUTE-EUROPE) marks an important step in this direction. The primary goal of the VF-QC is to provide a common structure for the growing quantum control community in Europe, for the promotion of quantum control and the provision of expertise to other scientific communities, to policy makers and the general public. Establishing common terminology, common standards and common visions are crucial prerequisites to maximizing the beneficial impact of quantum control methods on current and future technology, economics and society.

This strategic report has grown out of the Pan-European Coordination and support action on Optimal Control of Quantum Systems (QUAINT), funded by the European Commission under grant number 297861. We would like to thank the board of referees handling QUAINT and all colleagues who have participated in the community consultation for their comments and suggestions. In particular we acknowledge contributions from Tobias Brixner, Andreas Buchleitner, Daniel Burgarth, Simone Montangero, Enrico Prati, David Tannor, and Zdeněk Tošner. We are indebted to Per J. Liebermann for assisting the writing process as well as Frank Langbein, Peter K. Schuhmacher and Andrea Dumont for managing the community consultation.

\section{References}

1. The QUAINT website, www.quantumcontrol.eu (2015)

2. J.P. Dowling, G. Milburn, Phil. Trans. R. Soc. Lond. A 361, 1655 (2003)

3. M. Devoret, Quantum Machines: Measurement and Control of Engineered Quantum Systems, École de Physique des Houches 2011 (Oxford University Press, Oxford, 2014)

4. L.S. Pontryagin, V.G. Bol'tanskii, R.S. Gamkrelidze, E.F. Mischenko, The Mathematical Theory of Optimal Processes (Pergamon Press, New York, 1964)

5. E.B. Lee, L. Markus, Foundations of Optimal Control Theory (Wiley, New York, 1967)

6. A.E. Bryson, Y.C. Ho, Applied Optimal Control: Optimization, Estimation, and Control (Taylor and Francis, 1975)

7. R. Kalman, P.L. Falb, M.A. Arbib, Topics in Mathematical System Theory (McGraw-Hill, New York, 1969)

8. E. Sontag, Mathematical Control Theory (Springer, New York, 1998)
9. D.E. Kirk, Optimal Control Theory: An introduction (Dover Publications, New York, 2004)

10. J.M. Coron, Control and Nonlinearity (Am. Math. Soc., Providence RI, 2007)

11. A.A. Agrachev, Y.L. Sachkov, Control theory from the geometric viewpoint (Springer-Verlag, Berlin, 2004), Vol. 87

12. V. Jurdjevic, Geometric Control Theory (Cambridge University Press, Cambridge, 1997)

13. A. Bressan, B. Piccoli, Introduction to the mathematical theory of control, AIMS Series on Applied Mathematics (American Institute of Mathematical Sciences, Springfield, MO, 2007), Vol. 2

14. D. D'Alessandro, Introduction to Quantum Control and Dynamics, Applied Mathematics \& Nonlinear Science (Chapman and Hall, Boca Raton, 2008)

15. H.M. Wiseman, G.J. Milburn, Quantum Measurement and Control (Cambridge University Press, Cambridge, 2010)

16. S. Cong, Control of Quantum Systems: Theory and Methods (Wiley \& Sons, Singapore, 2014)

17. C. Brif, R. Chakrabarti, H. Rabitz, New J. Phys. 12, 075008 (2010)

18. G. Dirr, U. Helmke, GAMM-Mitt. 31, 59 (2008)

19. D. Dong, I.A. Petersen, IET Control Theory A. 4, 2651 (2010)

20. R. Zeier, T. Schulte-Herbrüggen, J. Math. Phys. 52, $113510(2011)$

21. C. Altafini, F. Ticozzi, IEEE Trans. Automat. Control 57, 1898 (2012)

22. A. Borzi, Nanoscale Syst. Math. Model. Theor. Appl. 1, $93(2012)$

23. B. Bonnard, D. Sugny, Optimal Control with Applications in Space and Quantum Dynamics, AIMS on Applied Mathematics (American Institute of Mathematical Sciences, Springfield, 2012), Vol. 5

24. A.G. Butkovskiy, Y.I. Samoilenko, Control of QuantumMechanical Processes and Systems (Kluwer Academic Publishers, Dordrecht, The Netherlands, 1990), (see also the translations from Russian originals: A.G. Butkovskiy and Yu. I. Samoilenko, Control of Quantum Systems, Part I and II, Autom. Remote Control (USSR) 40, pp. 485-502 and pp. 629-645 (1979), as well as: A. G. Butkovskiy and Yu. I. Samoilenko, Controllability of Quantum Objects, Dokl. Akad. Nauk. USSR 250, pp. 22-24 (1980).)

25. V. Jurdjevic, H. Sussmann, J. Differ. Eq. 12, 313 (1972)

26. H. Sussmann, V. Jurdjevic, J. Differ. Eq. 12, 95 (1972)

27. R.W. Brockett, SIAM J. Control 10, 265 (1972)

28. R.W. Brockett, SIAM J. Appl. Math. 25, 213 (1973)

29. D. Elliott, Bilinear Control Systems: Matrices in Action (Springer, London, 2009)

30. F. Albertini, D. D'Alessandro, IEEE Trans. Automat. Control 48, 1399 (2003)

31. I. Kurniawan, G. Dirr, U. Helmke, IEEE Trans. Automat. Contr. 57, 1984 (2012)

32. U. Boscain, M. Caponigro, T. Chambrion, M. Sigalotti, Commun. Math. Phys. 311, 423 (2012)

33. G. Turinici, in Mathematical Models and Methods for Ab Initio Quantum Chemistry (Springer Berlin Heidelberg, 2000), Lecture Notes in Chemistry, Vol. 74, pp. 75-92

34. S.G. Schirmer, H. Fu, A.I. Solomon, Phys. Rev. A 63, 063410 (2001)

35. C. Altafini, J. Math. Phys. 43, 2051 (2002) 
36. M. Mirrahimi, P. Rouchon, IEEE Trans. Automat. Control 49, 745 (2004)

37. J.M. Ball, J.E. Marsden, M. Slemrod, SIAM J. Control Optim. 20, 575 (1982)

38. K. Beauchard, J. Math. Pures Appl. 84, 851 (2005)

39. K. Beauchard, J. Coron, J. Funct. Anal. 232, 328 (2006)

40. K. Beauchard, C. Laurent, J. Math. Pures Appl. 94, 520 (2010)

41. T. Chambrion, P. Mason, M. Sigalotti, U. Boscain, Ann. Inst. Henri Poincaré 26, 329 (2009)

42. T. Chambrion, Automatica 48, 2040 (2012)

43. N. Boussaid, M. Caponigro, T. Chambrion, IEEE Trans. Automat. Control 58, 2205 (2013)

44. U. Boscain, M. Caponigro, M. Sigalotti, J. Differ. Eq. 256, 3524 (2014)

45. M. Keyl, R. Zeier, T. Schulte-Herbrüggen, New J. Phys. 16, $065010(2014)$

46. U. Boscain, P. Mason, G. Panati, M. Sigalotti, J. Math. Phys. 56, 092101 (2015)

47. K. Beauchard, J.M. Coron, P. Rouchon, Commun. Math. Phys. 296, 525 (2010)

48. J. Li, N. Khaneja, Phys. Rev. A 73, 030302 (2006)

49. J.S. Li, N. Khaneja, IEEE Trans. Automat. Control 54, $528(2009)$

50. Z. Leghtas, A. Sarlette, P. Rouchon, J. Phys. B 44, 154017 (2011)

51. G. Turinici, H. Rabitz, Phys. Rev. A 70, 063412 (2004)

52. K. Kobzar, T.E. Skinner, N. Khaneja, S. Glaser, B. Luy, J. Magn. Reson. 170, 236 (2004)

53. K. Kobzar, T.E. Skinner, N. Khaneja, S.J. Glaser, B. Luy, J. Magn. Reson. 194, 58 (2008)

54. T.E. Skinner, M. Braun, K. Woelk, N.I. Gershenzon, S.J. Glaser, J. Magn. Reson. 209, 282 (2011)

55. K. Kobzar, S. Ehni, T.E. Skinner, S.J. Glaser, B. Luy, J. Magn. Reson. 225, 142 (2012)

56. M. Goerz, E. Halperin, J.M. Aytac, C.P. Koch, K.B. Whaley, Phys. Rev. A 90, 032329 (2014)

57. C. Altafini, J. Math. Phys. 44, 2357 (2003)

58. G. Dirr, U. Helmke, I. Kurniawan, T. SchulteHerbrüggen, Rep. Math. Phys. 64, 93 (2009)

59. C. O'Meara, G. Dirr, T. Schulte-Herbrüggen, IEEE Trans. Automat. Contr. (IEEE-TAC) 57, 2050 (2012)

60. D. Reich, N. Katz, C. Koch, Sci. Rep. 5, 12430 (2015)

61. H.P. Breuer, E.M. Laine, J. Piilo, Phys. Rev. Lett. 103, 210401 (2009)

62. S. Lloyd, L. Viola, Phys. Rev. A 65, 010101 (2001)

63. V. Bergholm, T. Schulte-Herbrüggen, arXiv:1206.4945 (2012)

64. Z. Zimborás, R. Zeier, T. Schulte-Herbrüggen, D. Burgarth, Phys. Rev. A 92, 042309 (2015)

65. D. Burgarth, K. Maruyama, M. Murphy, S. Montangero, T. Calarco, F. Nori, M. Plenio, Phys. Rev. A 81, 040303 (2010)

66. D.K. Burgarth, P. Facchi, V. Giovanetti, H. Nakazato, S. Pascazio, K. Yuasa, Nat. Commun. 5, 5173 (2014)

67. R.E. Kalman, J. Basic Eng. 82, 35 (1960)

68. B. Bonnard, M. Chyba, Singular trajectories and their role in control theory, Mathematics and Applications (Springer-Verlag, Berlin, 2003), Vol. 40

69. U. Boscain, B. Piccoli, Optimal Synthesis for Control Systems on 2-D Manifolds, smai(Springer, 2004), Vol. 43

70. B. Piccoli, H. Sussmann, SIAM J. Control Optim. 39, $359(2000)$
71. N. Khaneja, S. Glaser, R. Brockett, Phys. Rev. A 65, 032301 (2002)

72. U. Boscain, P. Mason, J. Math. Phys. 47, 062101 (2006)

73. D. D'Alessandro, M. Dahled, IEEE Trans. Automat. Control 46, 866 (2001)

74. U. Boscain, G. Charlot, J.P. Gauthier, S. Guérin, H.R. Jauslin, J. Math. Phys. 43, 2107 (2002)

75. A. Garon, S.J. Glaser, D. Sugny, Phys. Rev. A 88, 043422 (2013)

76. B. Bonnard, M. Chyba, D. Sugny, IEEE Trans. Automat. Control 54, 2598 (2009)

77. M. Lapert, Y. Zhang, M. Braun, S.J. Glaser, D. Sugny, Phys. Rev. Lett. 104, 083001 (2010)

78. N. Khaneja, B. Luy, S.J. Glaser, Proc. Natl. Acad. Sci. USA 100, 13162 (2003)

79. N. Khaneja, J.S. Li, C. Kehlet, B. Luy, S. Glaser, Proc. Natl. Acad. Sci. USA 101, 14742 (2004)

80. D. Sugny, C. Kontz, H.R. Jauslin, Phys. Rev. A 76, 023419 (2007)

81. H. Yuan, C.P. Koch, P. Salamon, D. Tannor, Phys. Rev. A 85, 033417 (2012)

82. N. Khaneja, T. Reiss, B. Luy, S.J. Glaser, J. Magn. Reson. 162, 311 (2003)

83. T.O. Reiss, N. Khaneja, S.J. Glaser, J. Magn. Reson. 154, $192(2002)$

84. R.B. Wu, R. Long, J. Dominy, T.S. Ho, H. Rabitz, Phys. Rev. A 86, 013405 (2012)

85. H. Yuan, N. Khaneja, Phys. Rev. A 72, 040301 (2005)

86. H. Yuan, S.J. Glaser, N. Khaneja, Phys. Rev. A 76, $012316(2007)$

87. H. Yuan, N. Khaneja, Phys. Rev. A 84, 062301 (2011)

88. M. Lapert, Y. Zhang, M. Janich, S.J. Glaser, D. Sugny, Sci. Rep. 2, 589 (2012)

89. N. Khaneja, R. Brockett, S. Glaser, Phys. Rev. A 63 , 032308 (2001)

90. N. Khaneja, S.J. Glaser, Chem. Phys. 267, 11 (2001)

91. U. Boscain, T. Chambrion, J.P. Gauthier, J. Dynam. Control Syst. 8, 547 (2002)

92. N. Khaneja, T. Reiss, C. Kehlet, T. Schulte-Herbrüggen, S. Glaser, J. Magn. Reson. 172, 296 (2005)

93. P. de Fouquieres, S.G. Schirmer, S.J. Glaser, I. Kuprov, J. Magn. Reson. 212, 412 (2011)

94. S. Machnes, U. Sander, S.J. Glaser, P. de Fouquières, A. Gruslys, S. Schirmer, T. Schulte-Herbrüggen, Phys. Rev. A 84, 022305 (2011)

95. G. Ciaramella, A. Borzì, G. Dirr, D. Wachsmuth, SIAM J. Sci. Comput. 37, A319 (2015)

96. V.F. Krotov, Global Methods in Optimal Control (Marcel Dekker, New York, 1996)

97. Y. Maday, G. Turinici, J. Chem. Phys. 118, 8191 (2003)

98. D. Reich, M. Ndong, C. Koch, J. Chem. Phys. 136, 104103 (2012)

99. R. Eitan, M. Mundt, D. Tannor, Phys. Rev. A 83, 053426 (2011)

100. Z. Tošner, T. Vosegaard, C. Kehlet, N. Khaneja, S.J. Glaser, N. Nielsen, J. Magn. Reson. 197, 120 (2009)

101. H.J. Hogben, M. Krzystyniak, G.T.P. Charnock, P.J. Hore, I. Kuprov, J. Magn. Reson. 208, 179 (2011)

102. J.R. Johansson, P.D. Nation, F. Nori, Comput. Phys. Commun. 184, 1234 (2013)

103. J. Werschnik, E.K.U. Gross, J. Phys. B 40, 175 (2007)

104. T.E. Skinner, T.O. Reiss, B. Luy, N. Khaneja, S.J. Glaser, J. Magn. Reson. 167, 68 (2004) 
105. A. Walther, B. Julsgaard, L. Rippe, Y. Ying, S. Kröll, R. Fisher, S. Glaser, Phys. Scr. T 137, 014009 (2009)

106. M. Janich, R. Schulte, M.M. Schwaiger, S. Glaser, J. Magn. Reson. 213, 126 (2011)

107. F. Motzoi, J.M. Gambetta, S.T. Merkel, F.K. Wilhelm, Phys. Rev. A 84, 022307 (2011)

108. P.E. Spindler, Y. Zhang, B. Endeward, N. Gershernzon, T.E. Skinner, S. Glaser, T.F. Prisner, J. Magn. Reson. 218, 49 (2012)

109. D. Reich, J. Palao, C. Koch, J. Mod. Opt. 61, 822 (2014)

110. I.N. Hincks, C.E. Granade, T.W. Borneman, D.G. Cory, Phys. Rev. Appl. 4, 024012 (2015)

111. M. Lapert, R. Tehini, G. Turinici, D. Sugny, Phys. Rev. A 79, 063411 (2009)

112. A. Bartana, R. Kosloff, D.J. Tannor, J. Chem. Phys. 106, 1435 (1997)

113. S. Kallush, R. Kosloff, Phys. Rev. A 73, 032324 (2006)

114. T. Schulte-Herbrüggen, A. Spörl, N. Khaneja, S.J. Glaser, J. Phys. B 44, 154013 (2011)

115. R. Schmidt, A. Negretti, J. Ankerhold, T. Calarco, J.T. Stockburger, Phys. Rev. Lett. 107, 130404 (2011)

116. F. Floether, P. de Fouquieres, S. Schirmer, New J. Phys. 14, 073023 (2012)

117. M. Goerz, D. Reich, C. Koch, New J. Phys. 16, 055012 (2014)

118. T.E. Skinner, N.I. Gershenzon, J. Magn. Reson. 204, 248 (2010)

119. S. Meister, J.T. Stockburger, R. Schmidt, J. Ankerhold, J. Phys. A 47, 495002 (2014)

120. S. Machnes, D.J. Tannor, F.K. Wilhelm, E. Assémat, arXiv: $1507.04261(2015)$

121. D.V. Savostyanov, S.V. Dolgov, J.M. Werner, I. Kuprov, Phys. Rev. B 90, 085139 (2014)

122. P. Doria, T. Calarco, S. Montangero, Phys. Rev. Lett. 106, 190501 (2011)

123. T. Caneva, T. Calarco, S. Montangero, Phys. Rev. A 84, 022326 (2011)

124. V. Engel, C. Meier, D. Tannor, Local Control Theory: Recent Applications to Energy and Particle Transfer Processes in Molecules (John Wiley \& Sons, Inc., 2009), Vol. 141, pp. 29-101

125. M. Sugawara, J. Chem. Phys. 118, 6784 (2003)

126. X. Wang, S.G. Schirmer, IEEE Trans. Automat. Control 55, $2259(2010)$

127. A. Peirce, M. Dahleh, H. Rabitz, Phys. Rev. A 37, 4950 (1987)

128. S. Lloyd, S. Montangero, Phys. Rev. Lett. 113, 010502 (2014)

129. R. Chakrabarti, H. Rabitz, Int. Rev. Phys. Chem. 26, 671 (2007)

130. P. de Fouquieres, S.G. Schirmer, Infin. Dimens. Anal. Qu. 16, $1350021(2013)$

131. A.N. Pechen, D.J. Tannor, Phys. Rev. Lett. 106, 120402 (2011)

132. H. Rabitz, T.S. Ho, R. Long, R. Wu, C. Brif, Phys. Rev. Lett. 108, 198901 (2012)

133. A.N. Pechen, D.J. Tannor, Phys. Rev. Lett. 108, 198902 (2012)

134. G. Riviello, K. Moore Tibbetts, C. Brif, R. Long, R.B. Wu, T.S. Ho, H. Rabitz, Phys. Rev. A 91, 043401 (2015)

135. K. Moore Tibbetts, H. Rabitz, Phys. Chem. Chem. Phys. 17, $3164(2015)$
136. K. Bergmann, H. Theuer, B.W. Shore, Rev. Mod. Phys. 70, 1003 (1998)

137. N.V. Vitanov, T. Halfmann, B.W. Shore, K. Bergmann, Annu. Rev. Phys. Chem. 52, 763 (2001)

138. S. Guérin, H.R. Jauslin, Control of Quantum Dynamics by Laser Pulses: Adiabatic Floquet Theory (John Wiley \& Sons, Inc., 2003), Vol. 125, pp. 147-267

139. S. Teufel, Adiabatic Perturbation Theory in Quantum Dynamics (Springer-Verlag, Berlin, 2003)

140. R. Adami, U. Boscain, Controllability of the Schrödinger Equation via Intersection of Eigenvalues, in 2005 and 2005 European Control Conference. CDC-ECC '05. 44th IEEE Conference on Decision and Control (2005), pp. 1080-1085

141. U. Boscain, F. Chittaro, P. Mason, M. Sigalotti, IEEE Trans. Automat. Control 57, 1970 (2012)

142. U. Boscain, J.P. Gauthier, F. Rossi, M. Sigalotti, Commun. Math. Phys. 333, 1225 (2015)

143. E. Torrontegui, S. Ibáñez, S. Martínez-Garaot, M. Modugno, A. del Campo, D. Guéry-Odelin, A. Ruschhaupt, X. Chen, J.G. Muga, in Advances in Atomic, Molecular, and Optical Physics, edited by E. Arimondo, P.R. Berman, C.C. Lin (Academic Press, 2013), Vol. 62, pp. 117-169

144. A. Ruschhaupt, X. Chen, D. Alonso, J.G. Muga, New J. Phys. 14, 093040 (2012)

145. X. Chen, I. Lizuain, A. Ruschhaupt, D. Guéry-Odelin, J.G. Muga, Phys. Rev. Lett. 105, 123003 (2010)

146. D. Daems, A. Ruschhaupt, D. Sugny, S. Guérin, Phys. Rev. Lett. 111, 050404 (2013)

147. Y. Ban, X. Chen, E.Y. Sherman, J.G. Muga, Phys. Rev. Lett. 109, 206602 (2012)

148. A. Joye, Commun. Math. Phys. 275, 139 (2007)

149. M.S. Sarandy, D.A. Lidar, Phys. Rev. Lett. 95, 250503 (2005)

150. D.J. Egger, F.K. Wilhelm, Phys. Rev. Lett. 112, 240503 (2014)

151. H.P. Büchler, S. Diehl, A. Kantian, A. Micheli, P. Zoller, Phys. Rev. A 78, 042307 (2008)

152. F. Verstraete, M.M. Wolf, J.I. Cirac, Nat. Phys. 5, 633 (2009)

153. H. Krauter, C. Muschik, K. Jensen, W. Wasilewski, J. Petersen, J.I. Cirac, E. Polzik, Phys. Rev. Lett. 107, 080503 (2011)

154. S.G. Schirmer, X. Wang, Phys. Rev. A 81, 062306 (2010)

155. A. Pechen, H. Rabitz, J. Math. Sci. 199, 695 (2014)

156. L. Bouten, R. van Handel, M. James, SIAM J. Control Optim. 46, 2199 (2007)

157. K.R. Parthasarathy, An introduction to quantum stochastic calculus (Birkhauser, Basel, 1992)

158. M. Annunziato, A. Borzi, Math. Modelling Anal. 15, 393 (2010)

159. Y. Zhang, M. Lapert, D. Sugny, M. Braun, S.J. Glaser, J. Chem. Phys. 134, 054103 (2011)

160. A. Doherty, S. Habib, K. Jacobs, H. Mabuchi, S. Tan, Phys. Rev. A 62, 012105 (2000)

161. R. Judson, H. Rabitz, Phys. Rev. Lett. 68, 1500 (1992)

162. N. Yamamoto, Phys. Rev. X 4, 041029 (2014)

163. H.M. Wiseman, G.J. Milburn, Phys. Rev. Lett. 70, 548 (1993)

164. S. Lloyd, Phys. Rev. A 62, 022108 (2000)

165. L. Bouten, R. van Handel, M. James, SIAM Rev. 51, 239 (2009) 
166. J. Combes, K. Jacobs, Phys. Rev. Lett. 96, 010504 (2006)

167. J. Combes, H. Wiseman, K. Jacobs, Phys. Rev. Lett. 100, 160503 (2008)

168. I. Sayrin et al., Nature 477, 73 (2011)

169. G. Kirchmair, B. Vlastakis, Z. Leghtas, S. Nigg, H. Paik, M. Ginossar, E. Mirrahimi, L. Frunzio, S. Girvin, R. Schoelkopf, Nature 495, 205 (2013)

170. M.R. James, H.I. Nurdin, I.R. Petersen, IEEE Trans. Automat. Control 53, 1787 (2008)

171. J.E. Gough, S. Wildfeuer, Phys. Rev. A 80, 042107 (2009)

172. H. Mabuchi, Phys. Rev. A 78, 032323 (2008)

173. S. Iida, M. Yukawa, H. Yonezawa, N. Yamamoto, A. Furusawa, IEEE Trans. Automat. Control 57, 2045 (2012)

174. V. Buzek, M. Hillery, M. Ziman, M. Rosko, Quant. Inf. Proc. 5, 313 (2006)

175. M. Hillery, V. Buzek, Contemp. Phys. 50, 575 (2009)

176. J.E. Reiner, W.P. Smith, L.A. Orozco, H.M. Wiseman, J. Gambetta, Phys. Rev. A 70, 023819 (2004)

177. L. Martin, F. Motzoi, H. Li, M. Sarovar, B. Whaley, arXiv: 1506.07888 (2015)

178. D. Ristè, C.C. Bulting, K.W. Lehnert, L. DiCarlo, Phys. Rev. Lett. 109, 240502 (2012)

179. R. Vijay, C. Macklin, D.H. Slichter, S.J. Weber, K.W. Murch, R. Naik, A.N. Korotkov, I. Siddiqi, Nature 490 $77(2012)$

180. C. Emary, J. Gough, Phys. Rev. B 90, 205436 (2014)

181. D.J. Tannor, S.A. Rice, J. Chem. Phys. 83, 5013 (1985)

182. D. Tannor, R. Kosloff, S. Rice, J. Chem. Phys. 85, 5805 (1986)

183. R. Kosloff, S.A. Rice, P. Gaspard, S. Tersigni, D.J. Tannor, Chem. Phys. 139, 201 (1989)

184. S.A. Rice, M. Zhao, Optical control of molecular dynamics (John Wiley \& Sons, 2000)

185. P. Brumer, M. Shapiro, Principles and Applications of the Quantum Control of Molecular Processes (Wiley Interscience, 2003)

186. A.M. Weiner, Rev. Sci. Instrum. 71, 1929 (2000)

187. A. Monmayrant, S. Weber, B. Chatel, J. Phys. B 43, 103001 (2010)

188. R.J. Gordon, S.A. Rice, Annu. Rev. Phys. Chem. 48, 601 (1997)

189. H. Rabitz, R. de Vivie Riedle, M. Motzkus, K. Kompa, Science 288, 824 (2000)

190. R.J. Levis, G.M. Menkir, H. Rabitz, Science 292, 709 (2001)

191. C. Daniel, J. Full, L. González, C. Lupulescu, J. Manz, A. Merli, S. Vajda, L. Wöste, Science 299, 536 (2003)

192. T. Brixner, G. Gerber, Chem. Phys. Chem. 4, 418 (2003)

193. M. Dantus, V.V. Lozovoy, Chem. Rev. 104, 1813 (2004)

194. M. Wollenhaupt, V. Engel, T. Baumert, Ann. Rev. Phys. Chem. 56, 25 (2005)

195. P. Nuernberger, G. Vogt, T. Brixner, G. Gerber, Phys. Chem. Chem. Phys. 9, 2470 (2007)

196. Analysis and Control of Ultrafast Photoinduced Reactions, edited by O. Kühn, L. Wöste (Springer, Berlin, 2007)

197. K. Hoki, P. Brumer, Phys. Rev. Lett. 95, 168305 (2005)

198. M. Spanner, P. Brumer, Phys. Rev. A 73, 023809 (2006)

199. M. Spanner, P. Brumer, Phys. Rev. A 73, 023810 (2006)

200. P. Brumer, K. Hoki, M. Spanner, Isr. J. Chem. 47, 111 (2007)
201. T. Bayer, M. Wollenhaupt, C. Sarpe-Tudoran, T. Baumert, Phys. Rev. Lett. 102, 023004 (2009)

202. T. Bayer, M. Wollenhaupt, H. Braun, T. Baumert (2014), in Advances in Chemical Physics, 2015, 1-50, https://www. uni-kassel.de/fb10/institute/physik/ forschungsgruppen/femtosekundenspektroskopie/ veroeffentlichungen.html

203. C. Trallero-Herrero, D. Cardoza, T.C. Weinacht, J.L. Cohen, Phys. Rev. A 71, 013423 (2005)

204. C. Trallero-Herrero, J.L. Cohen, T. Weinacht, Phys. Rev. Lett. 96, 063603 (2006)

205. J.G. Underwood, M. Spanner, M.Y. Ivanov, J. Mottershead, B.J. Sussman, A. Stolow, Phys. Rev. Lett. 90, 223001 (2003)

206. B. Sussman, D. Townsend, M. Ivanov, A. Stolow, Science 314, 278 (2006)

207. T. Bayer, H. Braun, C. Sarpe, R. Siemering, P. von den Hoff, R. de Vivie-Riedle, T. Baumert, M. Wollenhaupt, Phys. Rev. Lett. 110, 123003 (2013)

208. D. Meshulach, Y. Silberberg, Nature 396, 239 (1998)

209. P. Brumer, M. Shapiro, Chem. Phys. 139, 221 (1989)

210. V.I. Prokhorenko, A.M. Nagy, S. Waschuk, L.S. Brown, R.R. Birge, R.D. Miller, Science 313, 1257 (2006)

211. P. van der Walle, M.T.W. Milder, L. Kuipers, J.L. Herek, Proc. Natl. Acad. Sci. USA 106, 7714 (2009)

212. G. Katz, M. Ratner, R. Kosloff, New J. Phys. 12, 015003 (2010)

213. M. Am-Shallem, R. Kosloff, J. Chem. Phys. 141, 044121 (2014)

214. U. Marvet, M. Dantus, Chem. Phys. Lett. 245, 393 (1995)

215. P. Gross, M. Dantus, J. Chem. Phys. 106, 8013 (1997)

216. W. Salzmann et al., Phys. Rev. Lett. 100, 233003 (2008)

217. P. Nuernberger, D. Wolpert, H. Weiss, G. Gerber, Proc. Natl. Acad. Sci. USA 107, 10366 (2010)

218. A. Merli et al., Phys. Rev. A 80, 063417 (2009)

219. L. Rybak, S. Amaran, M. Levin, L.and Tomza, R. Moszynski, R. Kosloff, C. Koch, Z. Amitay, Phys. Rev. Lett. 107, 273001 (2011)

220. C. Koch, M. Shapiro, Chem. Rev. 212, 4928 (2012)

221. S. Amaran, R. Kosloff, M. Tomza, R. Moszynski, L. Rybak, L. Levin, Z. Amitay, J.M. Berglund, D.M. Reich, C.P. Koch, J. Chem. Phys. 139, 164124 (2013)

222. L. Levin, W. Skomorowski, R. Kosloff, Z. Koch, C.P.and Amitay, Phys. Rev. Lett. 114, 233003 (2015)

223. H. Stapelfeldt, T. Seideman, Rev. Mod. Phys. 75, 543 (2003)

224. T. Seideman, E. Hamilton, Adv. At. Mol. Opt. Phys. 52, 289 (2006)

225. D. Sugny, A. Keller, O. Atabek, D. Daems, C.M. Dion, S. Guérin, H.R. Jauslin, Phys. Rev. A 69, 033402 (2004)

226. H. Katsuki, H. Chiba, B. Girard, C. Meier, K. Ohmori, Science 311, 1589 (2006)

227. M. Aeschlimann, M. Bauer, D. Bayer, T. Brixner, F.J. García de Abajo, W. Pfeiffer, M. Rohmer, C. Spindler, F. Steeb, Nature 446, 301 (2007)

228. W. Pfeiffer, M. Aeschlimann, T. Brixner, Optical Antennas (Cambridge University Press, 2013), pp. 135156, eds. M. Agio \& A. Alú

229. A. Bartana, R. Kosloff, D.J. Tannor, Chem. Phys. 267, 195 (2001)

230. D. Sofikitis, S. Weber, A. Fioretti, R. Horchani, M. Allegrini, B. Chatel, P. Comparat, D. Pillet, New J. Phys. 11, 055037 (2009) 
231. C.Y. Lien, C.M. Seck, Y.W. Lin, J.H.V. Nguyen, D.A. Tabor, B.C. Odom, Nat. Commun. 5, 4783 (2014)

232. D. Reich, C.P. Koch, New J. Phys. 15, 125028 (2013)

233. C. Koch, J.P. Palao, R. Kosloff, F. Masnou-Seeuws, Phys. Rev. A 70, 013402 (2004)

234. M.J. Wright, S.D. Gensemer, J. Vala, R. Kosloff, P.L. Gould, Phys. Rev. Lett. 95, 063001 (2005)

235. J. Danzl, E. Haller, M. Gustavsson, M. Mark, R. Hart, N. Bouloufa, O. Dulieu, H. Ritsch, H.C. Nägerl, Science 321, 1062 (2008)

236. K.K. Ni, S. Ospelkaus, M.H.G. de Miranda, A. Pe'er, B. Neyenhuis, J.J. Zirbel, S. Kotochigova, P.S. Julienne, D.S. Jin, J. Ye, Science 322, 231 (2008)

237. X. Li, C. Menzel-Jones, D. Avisar, M. Shapiro, Phys. Chem. Chem. Phys. 12, 15760 (2010)

238. D. Avisar, D.J. Tannor, Phys. Rev. Lett. 106, 170405 (2011)

239. D. Avisar, D.J. Tannor, J. Chem. Phys. 136, 214107 (2012)

240. J. Herek, M. Wohlleben, X. Cogdell, X. Zeidler, M. Motzkus, Nature 417, 533 (2002)

241. T. Caruso, S. Montangero, T. Calarco, S. Huelga, M. Plenio, Phys. Rev. A 85, 042331 (2012)

242. Y. Hover, F. Caruso, S. Montangero, T. Sarovar, T. Calarco, M.B. Plenio, K.B. Whaley, New J. Phys. 16, 045007 (2014)

243. N. Dudovich, D. Oron, Y. Silberberg, Nature 418, 512 (2002)

244. P. Hamm, M. Zanni, Concepts and Methods of 2D Infrared Spectroscopy (Cambridge University Press, 2011)

245. A.L. Serrano, J.S. Ghosh, A. Ostrander, M.T. Zanni, Opt. Express 23, 17815 (2015)

246. H. Frostig, T. Bayer, N. Dudovich, Y.C. Eldar, Y. Silberberg, Nat. Photon. 9, 339 (2015)

247. I. Pastirk, J. Dela-Cruz, K. Walowicz, V. Lozovoy, M. Dantus, Opt. Express 11, 1695 (2003)

248. J.M. Dela Cruz, I. Pastirk, M. Comstock, V.V. Lozovoy, M. Dantus, Proc. Natl. Acad. Sci. USA 101, 16996 (2004)

249. O. Katz, E. Small, Y. Bromberg, Y. Silberberg, Nat. Photon. 5, 372 (2011)

250. O. Katz, E. Small, Y. Silberberg, Nat. Photon. 6, 549 (2012)

251. D. Oron, E. Tal, Y. Silberberg, Opt. Express 13, 1468 (2005)

252. J. Ogilvie, D. Débarre, X. Solinas, J. Martin, E. Beaurepaire, M. Joffre, Opt. Express 14, 759 (2006)

253. R.S.C. Pillai, C. Boudoux, G. Labroille, N. Olivier, I. Veilleux, E. Farge, M. Joffre, E. Beaurepaire, Opt. Express 17, 12741 (2009)

254. J. Rehbinder, L. Brückner, A. Wipfler, T. Buckup, M. Motzkus, Opt. Express 22, 28790 (2014)

255. H. Li, D.A. Harris, B. Xu, P. Wrzesinski, V. Lozovoy, M. Dantus, Opt. Express 16, 5499 (2008)

256. F. Courvoisier, L. Bonacina, V. Boutou, L. Guyon, C. Bonnet, B. Thuillier, J. Extermann, M. Roth, H. Rabitz, J. Wolf, Farad. Discuss. 137, 37 (2008)

257. D. Kiselev, L. Bonacina, J. Wolf, Opt. Express 19, 24516 (2011)

258. A. Steinbacher, P. Nuernberger, T. Brixner, Phys. Chem. Chem. Phys. 17, 6340 (2015)

259. T. Matthews, I.R. Piletic, M. Selim, M. Simpson, W. Warren, Sci. Transl. Med. 3, 71ra15 (2011)
260. L. Englert, M. Wollenhaupt, L. Haag, C. Sarpe-Tudoran, B. Rethfeld, T. Baumert, Appl. Phys. A 92, 749 (2008)

261. T. Bohinski, K. Moore-Tibbetts, M. Tarazkar, D.A. Romanov, S. Matsika, R.J. Levis, J. Phys. Chem. Lett. 5, 4305 (2014)

262. K. Moore-Tibbetts, M. Tarazkar, T. Bohinski, D.A. Romanov, S. Matsika, R. Levis, J. Phys. B 48, 164002 (2015)

263. L. Greenman, C. Koch, K.B. Whaley, Phys. Rev. A 92, 013407 (2015)

264. I. Serban, J. Werschnik, E.K.U. Gross, Phys. Rev. A 71, $053810(2005)$

265. I. Schaefer, R. Kosloff, Phys. Rev. A 86, 063417 (2012)

266. D. Tannor, V. Kazakov, V. Orlov, in Time-dependent quantum molecular dynamics, edited by J. Broeckhove, L. Lathouwers (Plenum, 1992), pp. 347-360

267. J. Somlói, V.A. Kazakovski, D.J. Tannor, Chem. Phys. 172, 85 (1993)

268. P. Gross, D. Neuhauser, H. Rabitz, J. Chem. Phys. 96, $2834(1992)$

269. W. Zhu, J. Botina, H. Rabitz, J. Chem. Phys. 108, 1953 (1998)

270. Y.J. Yan, R.E. Gillilan, R.M. Whitnell, K.R. Wilson, S. Mukamel, J. Phys. Chem. 97, 2320 (1993)

271. J. Salomon, C. Dion, G. Turinici, J. Chem. Phys. 123, $144310(2005)$

272. M. Lapert, D. Sugny, Phys. Rev. A 85, 063418 (2012)

273. S. Ramakrishna, T. Seideman, Phys. Rev. Lett. 95, $113001(2005)$

274. M.Z. Hoque, M. Lapert, E. Hertz, F. Billard, D. Sugny, B. Lavorel, O. Faucher, Phys. Rev. A 84, 013409 (2011)

275. C. Gollub, M. Kowalewski, R. de Vivie Riedle, Phys. Rev. Lett. 101, 073002 (2008)

276. M. Schröder, A. Brown, New J. Phys. 11, 105031 (2009)

277. J. Palao, D.M. Reich, C.P. Koch, Phys. Rev. A 88, 053409 (2013)

278. A. Hansen, M. Sørensen, P. Staanum, M. Drewsen, Angew. Chem. Int. Ed. 51, 7960 (2012)

279. R. Rugango, J.E. Goeders, T.H. Dixon, J. Gray, N. Khanyile, G. Shu, R. Clark, K. Brown, New J. Phys. 17, 035009 (2015)

280. R. Ernst, G. Bodenhausen, A. Wokaun, Principles of Nuclear Magnetic Resonance in One and Two Dimensions (Clarendon Press Oxford, 1987)

281. A. Abragam, The Principles of Nuclear Magnetism (Oxford University Press, Oxford, 1961)

282. M.H. Levitt, Spin Dynamics: Basics of Nuclear Magnetic Resonance (Wiley, 2001)

283. A. Schweiger, G. Jeschke, Principles of Pulse Electron Paramagnetic Resonance (Oxford University Press, 2001)

284. M.A. Bernstein, K.F. King, Zhou, Handbook of MRI Pulse Sequences (Elsevier, Burlington-San DiegoLondon, 2004)

285. R. Ernst, Angew. Chem. Internat Ed. 31, 805 (1992)

286. K. Wüthrich, Angew. Chem. Int. Ed. 42, 3340 (2003)

287. P. Mansfield, Angew. Chem. Int. Ed. 43, 5456 (2004)

288. T. Kaufmann, J.M. Franck, T.J. Keller, R.P. Barnes, S.J. Glaser, J.M. Martinis, S. Han, J. Magn. Reson. 235, 95 (2013)

289. K. Kobzar, B. Luy, N. Khaneja, S.J. Glaser, J. Magn. Reson. 173, 229 (2005)

290. L.M.K. Vandersypen, I.L. Chuang, Rev. Mod. Phys. 76, 1037 (2005) 
291. C.A. Ryan, C. Negrevergne, M. Laforest, E. Knill, R. Laflamme, Phys. Rev. A 78, 012328 (2008)

292. J.A. Jones, Prog. Nucl. Magn. Reson. Spectrosc. 59, 91 (2011)

293. N.A. Kurnit, I.D. Abella, S.R. Hartmann, Phys. Rev. Lett. 13, 567 (1964)

294. F. Jelezko, J. Wrachtrup, Phys. Status Solidi A 203, 3207 (2006)

295. T. Häberle, D. Schmid-Lorch, K. Karrai, F. Reinhard, J. Wrachtrup, Phys. Rev. Lett. 111, 170801 (2013)

296. F. Dolde et al., Nat. Commun. 5, 3371 (2014)

297. A. Viale, S. Aime, Curr. Opin. Chem. Biol. 14, 90 (2010)

298. S.B. Duckett, R.E. Mewis, Acc. Chem. Res. 45, 1247 (2012)

299. L.T. Kuhn, Hyperpolarization Methods in NMR Spectroscopy (Springer, 2013)

300. A.W. Overhauser, Phys. Rev. 92, 411 (1953)

301. Q.Z. Ni, E. Daviso, T. Can, E. Markhasin, S. Jawla, T. Swager, R. Temkin, J. Herzfeld, R. Griffin, Acc. Chem. Res. 46, 1933 (2013)

302. C. Griesinger, M. Bennati, H.M. Vieth, C. Luchinat, G. Parigi, P. Höfer, F. Engelke, S.J. Glaser, V. Denysenkov, T.F. Prisner, Prog. Nucl. Magn. Reson. Spectrosc. 64, 4 (2012)

303. C.R. Bowers, D.P. Weitekamp, Phys. Rev. Lett. 57, 2645 (1986)

304. F. Jelezko, T. Gaebel, I. Popa, M. Domhan, A. Gruber, J. Wrachtrup, Phys. Rev. Lett. 93, 130501 (2004)

305. C. Müller et al., Nat. Commun. 5, 4703 (2014)

306. D.D. Awschalom, L.C. Bassett, A.S. Dzurak, E.L. Hu, J.R. Petta, Science 339, 1174 (2013)

307. J.J. Pla, K.Y. Tan, J.P. Dehollain, W.H. Lim, J.J.L. Morton, F.A. Zwanenburg, D.N. Jamieson, A.S. Dzurak, A. Morello, Nature 496, 334 (2013)

308. E.L. Hahn, Phys. Rev. 80, 580 (1950)

309. U. Haeberlen, The Principles of Nuclear Magnetism (Academic Press, New York, 1976)

310. M.H. Levitt, Prog. Nucl. Magn. Reson. Spectrosc. 18, 61 (1986)

311. M.H. Levitt, Composite pulses (Wiley, 1996)

312. R. Freeman, Prog. Nucl. Magn. Reson. Spectrosc. 32, 59 (1998)

313. T.O. Reiss, N. Khaneja, S.J. Glaser, J. Magn. Reson. 165, $95(2003)$

314. N. Khaneja, B. Heitmann, A. Spörl, H. Yuan, T. SchulteHerbrüggen, S.J. Glaser, Phys. Rev. A 75, 012322 (2007)

315. D. Stefanatos, N. Khaneja, S.J. Glaser, Phys. Rev. A 69 , 022319 (2004)

316. M. Lapert, E. Assémat, S.J. Glaser, D. Sugny, Phys. Rev. A 90, 023411 (2014)

317. M. Lapert, E. Assémat, S.J. Glaser, D. Sugny, J. Chem. Phys. 142, 044202 (2015)

318. J.B. Murdoch, A.H. Lent, M.R. Kritzer, J. Magn. Reson. 74, 226 (1987)

319. S.J. Glaser, G. Drobny, Chem. Phys. Lett. 164, 456 (1989)

320. H. Liu, S.J. Glaser, G. Drobny, J. Chem. Phys. 93, 7543 (1990)

321. R. Freeman, X. Wu, J. Magn. Reson. 75, 184 (1987)

322. R. Freeman, Chem. Rev. 91, 1397 (1991)

323. T. Untidt, T. Schulte-Herbrüggen, B. Luy, S. Glaser, C. Griesinger, O. Sorensen, N. Nielsen, Mol. Phys. 95, $787(1998)$
324. S. Conolly, D. Nishimura, A. Macovski, IEEE Trans. Med. Imag. MI-5, 106 (1986)

325. J. Mao, T.H. Mareci, K.N. Scott, E.R. Andrew, J. Magn. Reson. 70, 310 (1986)

326. D. Rosenfeld, Y. Zur, Magn. Reson. Med. 36, 401 (1996)

327. J.S. Li, J. Ruths, T.Y. Yu, H. Arthanari, G. Wagner, Proc. Natl. Acad. Sci. USA 108, 1879 (2011)

328. L.J. Edwards, D.V. Savostyanov, Z.T. Welderufael, D. Lee, I. Kuprov, J. Magn. Reson. 243, 107 (2014)

329. G. Matson, Magn. Reson. Imag. 12, 1205 (1994)

330. H. Liu, G. Matson, Magn. Reson. Med. 66, 1254 (2011)

331. S.J. Glaser, G.P. Drobny, Adv. Magn. Opt. Reson. 14, 35 (1990)

332. K. Kobzar, Ph.D. thesis, Technische Universität München (TUM), 2007

333. S. Ehni, Ph.D. thesis, Karlsruhe Institute of Technology, 2013

334. S.A. Smith, T.O. Levante, B.H. Meier, R.R. Ernst, J. Magn. Reson. 106, 75 (1994)

335. M. Veshtort, R.G. Griffin, J. Magn. Reson. 178, 248 (2006)

336. D.J. Tannor, A. Bartana, J. Phys. Chem. A 103, 10359 (1999)

337. U. Boscain, T. Chambrion, G. Charlot, Discrete Contin. Dyn. Syst. Ser. B 5, 957 (2005)

338. M. Lapert, Y. Zhang, S.J. Glaser, D. Sugny, J. Phys. B 44, 154014 (2011)

339. A.D. Boozer, Phys. Rev. A 85, 013409 (2012)

340. V. Mukherjee, A. Carlini, A. Mari, T. Caneva, M. S., T. Carlarco, R. Fazio, V. Giovannetti, Phys. Rev. A 88, 062326 (2013)

341. M. Lapert, E. Assémat, S.J. Glaser, D. Sugny, Phys. Rev. A 88, 033407 (2013)

342. F. Albertini, D. D'Alessandro, J. Math. Phys. 56, 012106 (2015)

343. T.E. Skinner, N.I. Gershenzon, M. Nimbalkar, W. Bermel, B. Luy, S.J. Glaser, J. Magn. Reson. 216, 78 (2012)

344. N.I. Gershenzon, T.E. Skinner, B. Brutscher, N. Khaneja, M. Nimbalkar, B. Luy, S. Glaser, J. Magn. Reson. 192, 235 (2008)

345. N.I. Gershenzon, K. Kobzar, B. Luy, S. Glaser, T. Skinner, J. Magn. Reson. 188, 330 (2007)

346. T.E. Skinner, K. Kobzar, B. Luy, M.R. Bendall, W. Bermel, N. Khaneja, S.J. Glaser, J. Magn. Reson. 179, 241 (2006)

347. T.E. Skinner, T.O. Reiss, B. Luy, N. Khaneja, S.J. Glaser, J. Magn. Reson. 172, 17 (2005)

348. T.E. Skinner, T. Reiss, B. Luy, N. Khaneja, S.J. Glaser, J. Magn. Reson. 163, 8 (2003)

349. E. Assémat, M. Lapert, Y. Zhang, M. Braun, S.J. Glaser, D. Sugny, Phys. Rev. A 82, 013415 (2010)

350. E. Assémat, L. Attar, M.J. Penouilh, M. Picquet, A. Tabard, Y. Zhang, S.J. Glaser, D. Sugny, Chem. Phys. 405, $71(2012)$

351. C.H. Bennett, I. Cirac, M.S. Leifer, D.W. Leung, N. Linden, S. Popescu, G. Vidal, Phys. Rev. A 66, 012305 (2002)

352. G. Vidal, K. Hammerer, J.I. Cirac, Phys. Rev. Lett. 88, 237902 (2002)

353. K. Hammerer, G. Vidal, J.I. Cirac, Phys. Rev. A 66, $062321(2002)$ 
354. N. Khaneja, F. Kramer, S.J. Glaser, J. Magn. Reson. 173 , $116(2005)$

355. S. Ehni, B. Luy, J. Magn. Reson. 232, 7 (2013)

356. N. Khaneja, S.J. Glaser, Phys. Rev. A 66, 060301 (2002)

357. S. Bose, Phys. Rev. Lett. 91, 207901 (2003)

358. R. Zeier, M. Grassl, T. Beth, Phys. Rev. A 70, 032319 (2004)

359. D. Stefanatos, N. Khaneja, S.J. Glaser, Phys. Rev. A 72, $062320(2005)$

360. T. Schulte-Herbrüggen, A.K. Spörl, N. Khaneja, S.J. Glaser, Phys. Rev. A 72, 042331 (2005)

361. A. Carlini, A. Hosoya, T. Koike, Y. Okudaira, Phys. Rev. Lett. 96, 060503 (2006)

362. H. Yuan, R. Zeier, N. Khaneja, Phys. Rev. A 77, 032340 (2008)

363. S.G. Schirmer, P.J. Pemberton-Ross, Phys. Rev. A 80 , 030301 (2009)

364. X. Wang, A. Bayat, S. Bose, S. Schirmer, Phys. Rev. A 82, $012330(2010)$

365. X. Wang, A. Bayat, S.G. Schirmer, S. Bose, Phys. Rev. A 81, $032312(2010)$

366. A. Carlini, A. Hosoya, T. Koike, Y. Okudaira, J. Phys. A 44, $145302(2011)$

367. M. Lapert, J. Salomon, D. Sugny, Phys. Rev. A 85 , $033406(2012)$

368. A. Carlini, T. Koike, Phys. Rev. A 86, 054302 (2012)

369. M. Nimbalkar, R. Zeier, J.L. Neves, H. Elavarasi, S. B. Yuan, N. Khaneja, K. Dorai, S.J. Glaser, Phys. Rev. A 85, 012325 (2012)

370. A. Carlini, T. Koike, J. Phys. A 46, 045307 (2013)

371. B. Bonnard, O. Cots, N. Shcherbakova, Math. Control Relat. Fields 3, 287 (2013)

372. B. Bonnard, O. Cots, J.B. Pomet, N. Shcherbakova, ESAIM Control Optim. Calc. Var. 20, 864 (2014)

373. H. Yuan, D. Wei, Y. Zhang, S. Glaser, N. Khaneja, Phys. Rev. A 89, 042315 (2014)

374. L. Van Damme, R. Zeier, S.J. Glaser, D. Sugny, Phys. Rev. A 90, 013409 (2014)

375. S. Ehni, B. Luy, J. Magn. Reson. 247, 111 (2014)

376. M. Nimbalkar, B. Luy, T.E. Skinner, J.L. Neves, N.I. Gershenzon, K. Kobzar, W. Bermel, S.J. Glaser, J. Magn. Reson. 228, 16 (2013)

377. J.L. Neves, B. Heitmann, N. Khaneja, S.J. Glaser, J. Magn. Reson. 201, 7 (2009)

378. F. Schilling, N.I. Warner, T.E. Gershenzon, M. Sattler, S.J. Glaser, Angew. Chem. Int. Ed. 53, 4475 (2014)

379. F. Schilling, S.J. Glaser, J. Magn. Reson. 223, 207 (2012)

380. G. Zhang, F. Schilling, S.J. Glaser, C. Hilty, Anal. Chem. 85, 2875 (2013)

381. V.D.M. Koroleva, S. Mandal, Y.Q. Song, M.D. Hürlimann, J. Magn. Reson. 230, 64 (2013)

382. T.W. Borneman, M.D. Hürlimann, D.G. Cory, J. Magn. Reson. 207, 220 (2010)

383. S. Mandal, T.W. Borneman, V.D.M. Koroleva, M.D. Hürlimann, J. Magn. Reson. 247, 54 (2014)

384. M. Braun, S.J. Glaser, J. Magn. Reson 207, 114 (2010)

385. M. Braun, S.J. Glaser, New J. Phys. 16, 115002 (2014)

386. A. Enthart, J.C. Freudenberger, J. Furrer, H. Kessler, B. Luy, J. Magn. Reson. 192, 314 (2008)

387. P. Tzvetkova, S. Simova, B. Luy, J. Magn. Reson. 186, $193(2007)$

388. T. Reinsperger, B. Luy, J. Magn. Reson. 239, 110 (2014)
389. D. Schulze-Suenninghausen, J. Becker, B. Luy, J. Am. Chem. Soc. 136, 1242 (2014)

390. C. Kehlet, A.C. Sivertsen, M. Bjerring, T.O. Reiss, N. Khaneja, S.J. Glaser, N.C. Nielsen, J. Am. Chem. Soc. 126, 10202 (2004)

391. T. Vosegaard, C. Kehlet, N. Khaneja, S.J. Glaser, N.C. Nielsen, J. Am. Chem. Soc. 127, 13768 (2005)

392. Z. Tosner, S.J. Glaser, N. Khaneja, N.C. Nielsen, J. Chem. Phys. 125, 184502/1 (2006)

393. J.S. Lee, R.R. Regatte, A. Jerschow, J. Chem. Phys. 129, 224510 (2008)

394. A.W. MacGregor, L.A. O’Dell, R.W. Schurko, J. Magn. Reson. 208, 103 (2011)

395. T. Yi-Peng, N. Xin-Fang, L. Jun, C. Hong-Wei, Z. XianYi, P. Xin-Hua, D. Jiang-Feng, Chin. Phys. Lett. 29, $127601(2012)$

396. N.M. Loening, B.J. van Rossum, H. Oschkinat, Magn. Reson. Chem. 50, 284 (2012)

397. D. Xu, K.F. King, Y. Zhu, G.C. McKinnon, Z.P. Liang, Magn. Reson. Med. 59, 547 (2008)

398. W.A. Grissom, D. Xu, A.B. Kerr, J.A. Fessler, IEEE Trans. Med. Imag. 28, 1548 (2009)

399. A. Massire, M.A. Cloos, A. Vignaud, D. Le Bihan, A. Amadon, N. Boulant, J. Magn. Reson. 230, 76 (2013)

400. M.S. Vinding, I.I. Maximov, Z. Tošner, N.C. Nielsen, J. Chem. Phys. 137, 054203 (2012)

401. T.E. Skinner, N.I. Gershenzon, M. Nimbalkar, S.J. Glaser, J. Magn. Reson. 217, 53 (2012)

402. M. Janich, M. McLean, R. Noeske, S. Glaser, R.F. Schulte, J. Magn. Reson. 223, 129 (2012)

403. G. Rancan, T.T. Nguyen, S.J. Glaser, J. Magn. Reson. 252, 1 (2015)

404. I. Maximov, Z. Tosner, N.C. Nielsen, J. Chem. Phys. 128, 184505 (2008)

405. N. Pomplun, B. Heitmann, N. Khaneja, S.J. Glaser, Appl. Magn. Reson. 34, 331 (2008)

406. N. Pomplun, S.J. Glaser, Phys. Chem. Chem. Phys. 12, 5791 (2010)

407. Y. Hovav, A. Feintuch, S. Vega, D. Goldfarb, J. Magn. Reson. 238, 94 (2014)

408. C. Bretschneider, A. Karabanov, N. Nielsen, W. Köckenberger, J. Chem. Phys. 136, 094201 (2012)

409. S.S. Köcher, T. Heydenreich, S.J. Glaser, J. Magn. Reson. 249, 63 (2014)

410. I. Kuprov, J. Magn. Reson. 233, 107 (2013)

411. A. Garon, R. Zeier, S. Glaser, Phys. Rev. A 91, 042122 (2015)

412. N. Khaneja, Phys. Rev. A 76, 032326 (2007)

413. J.S. Hodges, J.C. Yang, C. Ramanathan, D.G. Cory, Phys. Rev. A 78, 010303 (2008)

414. A. Dementyev, D.G. Cory, C. Ramanathan, Phys. Rev. B 77, 024413 (2008)

415. S. van Frank, A. Negretti, T. Berrada, R. Bücker, S. Montangero, J.F. Schaff, T. Schumm, T. Calarco, J. Schmiedmayer, Nat. Commun. 5, 4009 (2014)

416. S. Rosi, A. Bernard, N. Fabbri, L. Fallani, C. Fort, M. Inguscio, T. Calarco, S. Montangero, Phys. Rev. A 88, 021601 (2013)

417. N. Timoney, V. Elman, S. Glaser, C. Weiss, M. Johanning, W. Neuhauser, C. Wunderlich, Phys. Rev. A 77, 052334 (2008)

418. J. Scheuer et al., New J. Phys. 16, 093022 (2014) 
419. F. Motzoi, J.M. Gambetta, P. Rebentrost, F.K. Wilhelm, Phys. Rev. Lett. 103, 110509 (2009)

420. J.M. Chow, L. DiCarlo, J.M. Gambetta, F. Motzoi, L. Frunzio, S.M. Girvin, R.J. Schoelkopf, Phys. Rev. A 82, 040305 (2010)

421. E. Lucero et al., Phys. Rev. A 82, 042339 (2010)

422. R. Schutjens, F.A. Dagga, D.J. Egger, F.K. Wilhelm, Phys. Rev. A 88, 052330 (2013)

423. V. Vesterinen, O.P. Saira, A. Bruno, L. DiCarlo (2014), e-prints arXiv: 1405.0450

424. J. Kelly et al., Phys. Rev. Lett. 112, 240504 (2014)

425. J.T. Muhonen et al., J. Phys: Condens. Matter 27, 154205 (2015)

426. J.J. Pla, K.Y. Tan, J.P. Dehollain, W.H. Lim, J.J. Morton, D.N. Jamieson, A. Morello, Nature 489, 541 (2012)

427. E. Kawakami, P. Scarlino, D.R. Ward, F.R. Braakman, D.E. Savage, M.G. Lagally, L.M.K. Vandersypen, Nat. Nanotechnol. 9, 666 (2014)

428. J.R. Petta, A.C. Johnson, J.M. Taylor, E.A. Laird, A. Yacoby, M.D. Lukin, C.M. Marcus, A.C. Gossard, Science 309, 2180 (2005)

429. B.M. Maune, M.G. Borselli, B. Huang, T.D. Ladd, P.W. Deelman, K.S. Holabird, A.T. Hunter, Nature 481, 344 (2012)

430. D. Kim, Z. Shi, C.B. Simmonds, D.R. Ward, J.R. Prance, T.S. Koh, M.A. Eriksson, Nature 511, 70 (2014)

431. A.D. Greentree, J.H. Cole, A.R. Hamilton, L.C.L. Hollenberg, Phys. Rev. B 70, 235317 (2004)

432. E. Ferraro, M. De Michielis, M. Fanciulli, E. Prati, Phys. Rev. B 91, 075435 (2015)

433. F.R. Braakman, P. Barthelmy, C. Reichl, W. Wegscheider, L.M.K. Vandersypen, Nat. Nanotechnol. 8, 432 (2013)

434. B.E. Anderson, H. Sosa-Martinez, C.A. Riofrío, I.H. Deutsch, P.S. Jessen, Phys. Rev. Lett. 114, 240401 (2015)

435. J.M. Cai, B. Naydenov, R. Pfeiffer, L.P. McGuinness, K.D. Jahnke, F. Jelezko, M.B. Plenio, A. Retzker, New J. Phys. 14, 113023 (2012)

436. G. De Chiara, T. Calarco, M. Anderlini, S. Montangero, P.J. Lee, B.L. Brown, W.D. Phillips, J.V. Porto, Phys. Rev. A 77, 052333 (2008)

437. K. Singer, U. Poschinger, M. Murphy, P. Ivanov, F. Ziesel, T. Calarco, F. Schmidt-Kaler, Rev. Mod. Phys. 82, 2609 (2010)

438. H.A. Fürst, M.H. Goerz, U.G. Poschinger, M. Murphy, S. Montangero, T. Calarco, F. Schmidt-Kaler, K. Singer, C.P. Koch, New J. Phys. 16, 075007 (2014)

439. T. Caneva, M. Murphy, T. Calarco, R. Fazio, S. Montangero, V. Giovannetti, G.E. Santoro, Phys. Rev. Lett. 103, 240501 (2009)

440. M. Murphy, S. Montangero, V. Giovannetti, T. Calarco, Phys. Rev. A 82, 022318 (2010)

441. A. Gorshkov, T. Calarco, M. Lukin, A. Sørensen, Phys. Rev. A 77, 043806 (2008)

442. J. Grond, J. Schmiedmayer, U. Hohenester, Phys. Rev. A 79, 021603 (2009)

443. R. Fisher, H. Yuan, A. Spörl, S.J. Glaser, Phys. Rev. A 79, 042304 (2009)

444. K. Rojan, D. Reich, I. Dotsenko, J. Raimond, C. Koch, G. Morigi, Phys. Rev. A 90, 023824 (2014)

445. T. Caneva, T. Calarco, R. Fazio, G. Santoro, S. Montangero, Phys. Rev. A 84, 012312 (2011)
446. T. Caneva, T. Calarco, S. Montangero, New J. Phys. 14, $093041(2012)$

447. T. Calarco, U. Dorner, P.S. Julienne, C.J. Williams, P. Zoller, Phys. Rev. A 70, 012306 (2004)

448. U. Dorner, T. Calarco, P. Zoller, P. Grangier, J. Opt. B 7, S341 (2005)

449. P. Treutlein, T.W. Hänsch, J. Reichel, A. Negretti, M.A. Cirone, T. Calarco, Phys. Rev. A 74, 022312 (2006)

450. M. Cozzini, T. Calarco, A. Recati, P. Zoller, Opt. Commun. 264, 375 (2006)

451. M. Goerz, T. Calarco, C. Koch, J. Phys. B 44, 154011 (2011)

452. U. Poulsen, S. Sklarz, D. Tannor, T. Calarco, Phys. Rev. A 82, 012339 (2010)

453. H. Doerk, Z. Idziaszek, T. Calarco, Phys. Rev. A 81, $012708(2010)$

454. G. Waldherr et al., Nature 506, 204 (2014)

455. J. Clarke, F.K. Wilhelm, Nature 453, 1031 (2008)

456. S. Montangero, T. Calarco, R. Fazio, Phys. Rev. Lett. 99, 170501 (2007)

457. A. Spörl, T. Schulte-Herbrüggen, S.J. Glaser, V. Bergholm, M.J. Storcz, J. Ferber, F.K. Wilhelm, Phys. Rev. A 75, 012302 (2007)

458. H. Jirari, F.W.J. Hekking, O. Buisson, Europhys. Lett. 87, 28004 (2009)

459. A. Cross, J. Gambetta, Phys. Rev. A 91, 032325 (2015)

460. D.J. Egger, F.K. Wilhelm, Supercond. Sci. Technol. 27, 014001 (2014)

461. P. Rebentrost, I. Serban, T. Schulte-Herbrüggen, F.K. Wilhelm, Phys. Rev. Lett. 102, 090401 (2009)

462. D.J. Egger, F.K. Wilhelm, Phys. Rev. A 90, 052331 (2014)

463. M.M. Müller, U. Poschinger, T. Calarco, F. Montangnero, S. Schmidt-Kaler, Phys. Rev. A 92, 053423 (2015)

464. G. Jäger, U. Hohenester, Phys. Rev. A 88, 035601 (2013)

465. M. Möttönen, R. de Sousa, J. Zhang, K.B. Whaley, Phys. Rev. A 73, 022332 (2006)

466. T. Rybár, S.N. Filippov, M. Ziman, V. Buzek, J. Phys. B 45, 154006 (2012)

467. G. Passante, O. Moussa, C. Ryan, R. Laflamme, Phys. Rev. Lett. 103, 250501 (2009)

468. R. Marx, A.F. Fahmy, L. Kauffman, S. Lomonaco, A. Spörl, N. Pomplun, T. Schulte-Herbrüggen, J. Myers, S. Glaser, Phys. Rev. A 81, 032319 (2010)

469. T. Schulte-Herbrüggen, R. Marx, A. Fahmy, L. Kauffman, S. Lomonaco, N. Khaneja, S.J. Glaser, Phil. Trans. R. Soc. A 370, 4651 (2012)

470. J.P. Palao, R. Kosloff, Phys. Rev. Lett. 89, 188301 (2002)

471. J.P. Palao, R. Kosloff, Phys. Rev. A 68, 062308 (2003)

472. C.M. Tesch, R. de Vivie-Riedle, Phys. Rev. Lett. 89, $157901(2002)$

473. V. Mukherjee, V. Giovannetti, R. Fazio, S. Huelga, T. Carlarco, M. S., New J. Phys. 17, 063031 (2015)

474. M.D. Grace, J. Dominy, R. Kosut, C. Brif, H. Rabitz, New J. Phys. 12, 015001 (2010)

475. M.M. Müller, D.M. Reich, M. Murphy, H. Yuan, J. Vala, K.B. Whaley, T. Calarco, C.P. Koch, Phys. Rev. A 84, 042315 (2011)

476. P. Watts, J. Vala, M.M. Müller, T. Calarco, K.B. Whaley, D.M. Reich, M.H. Goerz, C.P. Koch, Phys. Rev. A 91, 062306 (2015) 
477. M.H. Goerz, G. Gualdi, D.M. Reich, C.P. Koch, F. Motzoi, K.B. Whaley, J. Vala, M.M. Müller, S. Montangero, T. Calarco, Phys. Rev. A 91, 062307 (2015)

478. F. Platzer, F. Mintert, A. Buchleitner, Phys. Rev. Lett. 105, 020501 (2010)

479. S. Sklarz, D. Tannor, Phys. Rev. A 66, 053619 (2002)

480. U. Hohenester, P.K. Rekdal, A. Borzì, J. Schmiedmayer, Phys. Rev. A 75, 023602 (2007)

481. G. Jäger, D. Reich, M.H. Goerz, C.P. Koch, U. Hohenester, Phys. Rev. A 90, 033628 (2014)

482. T. Schulte-Herbrüggen, A.K. Spörl, K. Waldherr, T. Gradl, S.J. Glaser, T. Huckle, in High-Performance Computing in Science and Engineering, Garching 2007 (Springer, Berlin, 2008), pp. 517-533, (an extended version: http://arXiv.org/pdf/0712.3227)
483. A.G. Fowler, J.M. Martinis, Phys. Rev. A 89, 032316 (2014)

484. R. Barends et al., Nature 508, 500 (2014)

485. J. Preskill, e-prints arXiv:1203.5813, 25th Solvay Conference on Physics, 19-22 October 2011. (2012)

486. G. Kurizki, P. Bertet, Y. Kubo, K. Mølmer, D. Petrosyan, P. Rabl, J. Schmiedmayer, Proc. Natl. Acad. Sci. USA 112, 3866 (2015)

Open Access This is an open access article distributed under the terms of the Creative Commons Attribution License (http://creativecommons.org/licenses/by/4.0), which permits unrestricted use, distribution, and reproduction in any medium, provided the original work is properly cited. 